\title{
Programmable Active Mirror: A Scalable Decentralized Router
}

\author{
Austin Fikes $\left.^{(}\right)$, Graduate Student Member, IEEE, Parham P. Khial ${ }^{\circledR}$, Graduate Student Member, IEEE, \\ Samir Nooshabadi, Graduate Student Member, IEEE, and Ali Hajimiri ${ }^{\circledR}$, Fellow, IEEE
}

\begin{abstract}
This work proposes and demonstrates the scalable router array that eliminates the internal centralization of conventional arrays, unlocking scalability, and the potential for a system composed of spatially separated elements that do not share a common timing reference. Architectural variations are presented, and their specific tradeoffs are discussed. The general operation, steering capabilities, signal and noise considerations, and timing control advantages are evaluated through analysis, simulation, and measurements. An element-level CMOS radio frequency integrated circuit (RFIC) is developed and used to demonstrate a four-element $25 \mathrm{GHz}$ prototype router. The RFIC's programmable true time delay (TTD) control is used to correct path-length-difference-induced intersymbol interference (ISI) and improve a rerouted 270-Mb/s 64-QAM constellation from a completely scrambled state to an EVM of $4 \% \mathrm{rms}$ $(-28 \mathrm{~dB})$. The prototype scalable router's concurrent dual-beam capabilities are demonstrated by simultaneously steering two full power beams at 24.9 and $25 \mathrm{GHz}$ in two different directions in a free-space electromagnetic setup.
\end{abstract}

Index Terms-Aperture, integrated circuits, phased array, scalable relay, true time delay (TTD).

\section{INTRODUCTION}

I NSATIABLE demand for bandwidth is regulated by Shannon's theorem, which states that the maximum channel capacity is proportional to the available bandwidth and the logarithm of the signal-to-noise ratio (SNR) (plus 1). ${ }^{1}$ System and circuit designers have toiled to improve link budgets and achieve higher data rates and system capacities. On the circuit side, there has been a push to higher frequencies (where more bandwidth is available) by improving critical circuit building block performance, e.g., power amplifier efficiency and linearity or receiver sensitivity. On the system level, we have moved from single-channel static transmitters and receivers to sophisticated programmable massive multiple-input multipleoutput (MIMO) systems that can form larger apertures in transmitters and/or receivers to perform a slew of complex functions [1]-[3]. These arrays enhance the effective isotropic radiated power (EIRP) in transmitters and sensitivity of receivers to increase the available data rates through the second

\footnotetext{
${ }^{1} R_{B}=B \cdot \log 2(\mathrm{SNR}+1)$.

Manuscript received August 28, 2020; revised November 13, 2020; accepted November 16, 2020. Date of publication December 22, 2020; date of current version March 4, 2021. (Austin Fikes and Parham P. Khial contributed equally to this work.) (Corresponding author: Austin Fikes.)

The authors are with the Department of Electrical Engineering, California Institute of Technology, Pasadena, CA 91125 USA (e-mail: afikes@caltech.edu).

Color versions of one or more figures in this article are available at https://doi.org/10.1109/TMTT.2020.3042516.

Digital Object Identifier 10.1109/TMTT.2020.3042516
}

key parameter in Shannon's theorem, i.e., SNR. Also, the spatial directionality and beam confinement provided by arrays enable more effective spatial partitioning of the bandwidth, leading to higher frequency reuse ratios and smaller cell sizes.

Despite these clear advantages, existing phased arrays face aperture scaling limitations inherent to their centralized architecture. The challenge of aggregating every element's signals within the array grows perniciously at higher element numbers and aperture sizes. Furthermore, for high-speed data communication, the difference in data arrival (and departure) times between elements generates dispersion manifested as intersymbol interference (ISI) [4] that must be dealt with through array level delays or complex equalization schemes in a centralized fashion. Even clever designs rapidly reach practical limitations of signal routing density, interface bandwidth, and data synchronization. In addition, a centralized approach precludes spatially and/or electrically separated apertures working together.

In this article, we propose the scalable router: a decentralized relay array architecture that can selectively receive multiple signals from several desired incident angles and retransmit them in other arbitrary directions with minimal data distortion. Such scalable routers unite smaller spatially and electrically separated apertures to produce an effective large aperture at high data rates in a decentralized and dynamic fashion.

Intuitively, a scalable router is analogous to a mirror that can be dynamically reoriented in different directions for different incident signals that will bounce each one of those incoming beams toward different targets in various locations. Unlike a standard passive mirror, this programmable active one amplifies and conditions the signals it reflects. Furthermore, this mirror can be constructed out of multiple disconnected elements that could also move in real time.

The ability to operate multiple elements to form a decentralized, nonuniform, and/or dynamically changing array can open a plethora of new opportunities. For instance, locally powered arrays of elements with no need for timing reference synchronization can be deployed, gradually built-up, and constantly changed across unused walls, ceilings, and buildings surfaces at multiple locations (see Fig. 1). Furthermore, future infrastructure can allow such systems to be incorporated into various platforms, such as mobile devices, vehicles, building infrastructures, airborne systems, and satellites. It is obvious that such arrays would greatly benefit from architectures 

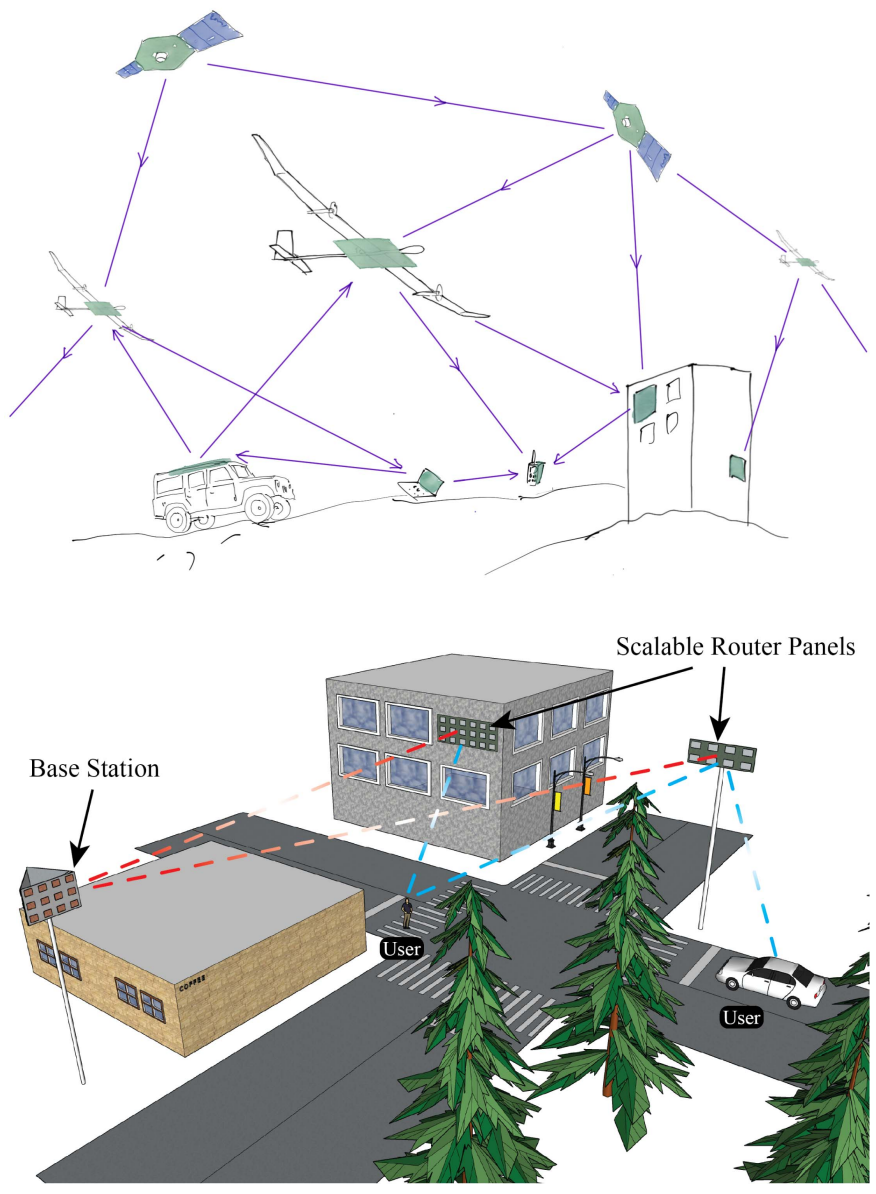

Fig. 1. Scalable router can be formed by apertures spread across a variety of static and moving surfaces. It can extend the effective reach of basestations to greater distances or areas blocked by obstructions.

that allow decentralized operation with dynamically moving elements.

In Section II, we present the evolution of the proposed idea and its high-level representation. This is followed by a detailed discussion of the various tradeoffs in such architectures in Section III. Details of an integrated circuit implementation are presented in Section IV followed by the measurement results of the ICs and an exemplary scalable relay implemented using the ICs in Section V. Section VI provides a summary and conclusion.

\section{Decentralized Distributed Router}

The scalable router architecture can be seen as the evolution and marriage of two existing microwave concepts: the bent pipe relay and the standard centralized phased array. The bent pipe relay is essentially a receive antenna, an amplifier, and a transmit antenna connected in series as shown in Fig. 2(a). This simple system reamplifies incident signals and redirects them with a static, unchanging radiation pattern.

A bent pipe relay can be modified to use two standard $M$ element phased arrays: one used as a receiver and the other

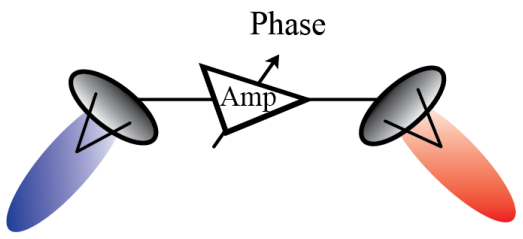

(a)

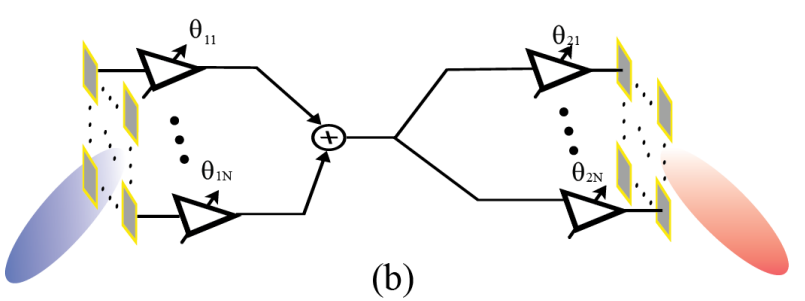

(b)

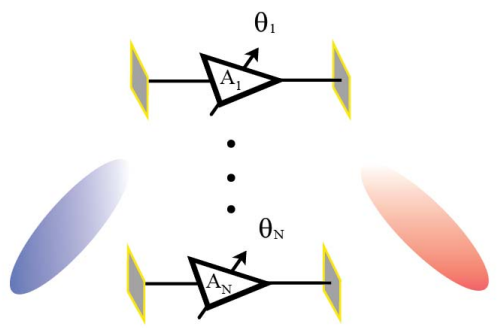

(c)

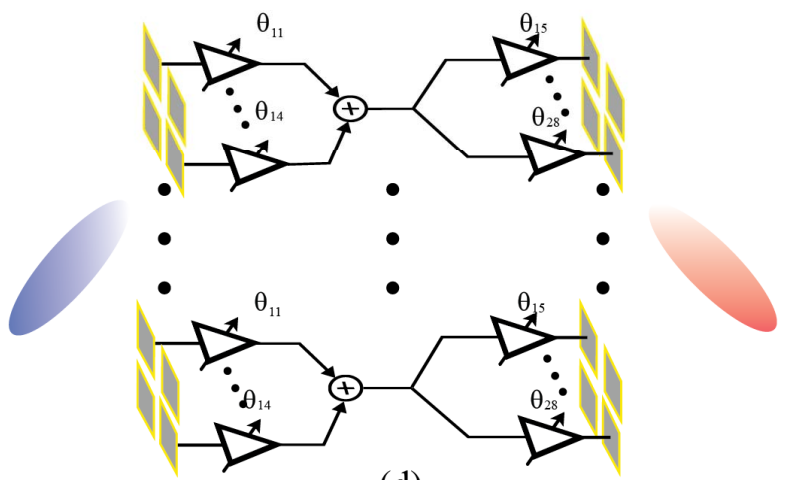

(d)

Fig. 2. (a) Bent pipe relay. (b) Centralized phased array implementation of bent pipe relay. (c) Scalable router system architecture. (d) Hybrid scalable router.

used as a transmitter as shown in Fig. 2(b). Each phased array creates an electronically steered beam of microwave power by controlling the phase of each element within an antenna array. Now, the system can electronically steer the transmit and receive beams.

To create the scalable router, we split the centralized aggregation node that is shared by all elements in the array and add tunable broadband time delays to each branch, as shown in the next step in the progression shown in Fig. 2(c). For an idealized, conceptual model in which amplification, summation, and delay are linear operations, this change is simply an application of the distributive property to a delayed array. Rather than sum and then split the incident signals within the system, signal summation occurs only in the reradiated beam. The tunable delay elements allow the receive and transmit 


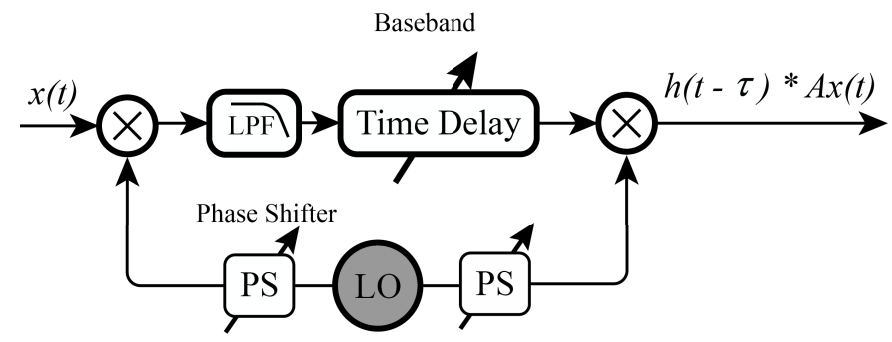

Fig. 3. Possible integrated circuit branch implementation using baseband time delay. A scalable router branch provides amplification, time delay, and filtering to the signal that it receives and transmits.

beams to be steered without centralized signal aggregation occurring within the router. ${ }^{2}$

While, at a highly abstracted level, the scalable router can perform all of the functions of a phased array relay, in a real system, there are profound implications for noise, linearity, isolation, and other system parameters that we will explore in this work. Fig. 2(d), described as a hybrid scalable router, represents a syncretic state of local centralization and system-level decentralization in which the router branches are composed of smaller phased arrays. The benefits offered by this array of arrays are described in Section III.

A critical feature of the scalable router is that each branch (receive element connected to a transmit element) does not interface with other branches within the array. The transmit and receive beams are steered entirely by setting the delay within each branch (possibly as low-frequency digital signals). Not only does this architecture bypass the challenge of centralizing data but also means that the branches can be implemented without a shared timing reference. The router is fully decentralized: a router may be formed by apertures that are physically separated. The decentralized operation of the scalable router emerges from each branch performing a decentralized function, not reliant on information from any other branch. Fig. 3 models the idealized operation of a single branch where amplification, delay, and filtering are performed. While these functions can be accomplished by systems at any frequency built with a variety of technologies, Fig. 3 shows a down-conversion/up-conversion implementation well-suited for integrated circuits at microwave frequencies. Interestingly, the decentralized architecture does not require the local oscillator (LO) signals within a branch to be phase or frequency locked to any other branch. Branch independence enables routers formed by a combination of static arrays, satellites, autonomous aircraft, ground vehicles, or any other surface that can support an aperture. Table I summarizes the differences between the scalable router architecture and conventional phased arrays.

The scalable router architecture is well suited for integration in emerging mm-wave communication infrastructure. Universal adoption of mm-wave systems is hindered by their

\footnotetext{
${ }^{2}$ There are some similarities (and differences) between the scalable router architecture and early space fed phased arrays [5]. The scalable router architecture and space fed arrays both have tunable elements with radiative inputs and outputs but differ as the scalable router is decentralized, uses true time delay (TTD), and operates in the radiative far-field.
}

TABLE I

Scalable Router Versus Conventional Phased Array

\begin{tabular}{|c||c|c|}
\hline & Scalable Router & $\begin{array}{c}\text { Conventional } \\
\text { Phased Array }\end{array}$ \\
\hline \hline Beam forming/steering & Carrier and data & Carrier only \\
\hline $\begin{array}{c}\text { Avoids internal, centralized } \\
\text { signal combining and } \\
\text { distribution }\end{array}$ & Yes & No \\
\hline Aperture scalability & Yes & Limited by ISI \\
\hline $\begin{array}{c}\text { Operate without elements } \\
\text { sharing timebase }\end{array}$ & Yes & No \\
\hline Fully decentralized & Yes & No \\
\hline
\end{tabular}

line-of-sight nature and the high absorption of walls and other obstructions at these frequencies. Attempting to overcome these issues in multiroom indoor settings, dense urban environments, remote areas, and flying systems using traditional phased arrays can lead to unwieldy apertures and power requirements (see Fig. 1). The scalable router excels in this niche. It can dynamically bounce signals around obstacles or extend and fortify low-quality communication links. The scalable decentralized router architecture can enable uninterrupted high-speed connectivity in the presence of large path loss and static and/or dynamic obstructions.

Several possible scalable router use-cases are examples of cooperative diversity, which is used as an umbrella term for multiantenna, relay-reliant, multiuser, or multihop schemes intended to increase channel capacity in communication networks [6]-[9]. A substantial body of theoretical cooperative diversity research exists, often focusing on optimizing the capacity of a hypothetical network consisting of a base station and several cellphone users given power constraints and incomplete channel state information [10]-[14]. While these analytical works have not explored the challenges, potential, and emergent capabilities of large-scale relay array hardware, such as the scalable router, their analysis might be fruitfully adapted for specific scalable router use scenarios.

\section{System Behavior AND PROPERTIES}

\section{A. Electronically Steerable Microwave Mirror}

The scalable router can act as an electronically steerable mirror at microwave frequencies. As the delay within each branch is electronically changed (mirror is rotated), the incident signal is conditioned and rerouted (reflected) to a new direction. Fig. 4 models the electronically steerable mirror analogy for a 16-element scalable router. Despite our desire for tidy comparisons, the mirror analogy elides subtle but critical aspects of scalable router beamforming.

The beam patterns of dynamic, spatially decentralized routers can be determined with a geometric derivation. Unlike a conventional centralized phased array, no aggregation occurs within the scalable router, intimately linking the receive and transmit gain beam patterns and deviating from the behavior predicted by our earlier geometric optics analogy.

Fig. 5(a) shows a general, decentralized array structure. The relationship between the intended direction of the received beam pattern, $\hat{R}_{r x}$, the intended direction of the transmit pattern, $\hat{R}_{t x}$, and the unwrapped phase (a surrogate for the 


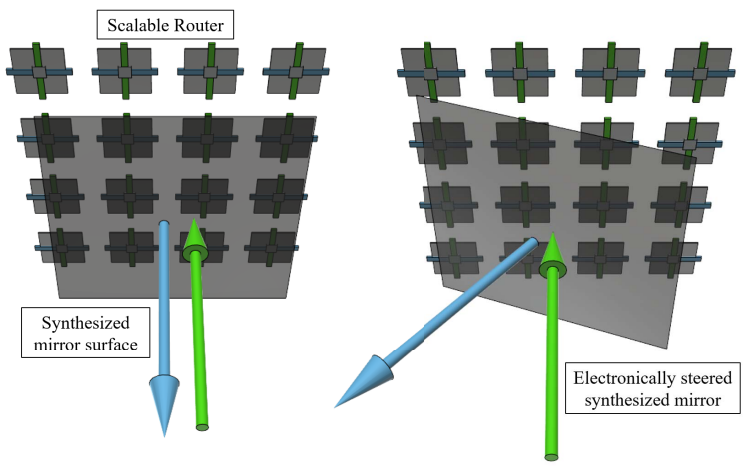

Fig. 4. 16-element scalable router synthesizes a microwave mirror (shown as transparent) that can be electronically steered.

delay), $\delta_{m}$, of each branch at location $\vec{r}_{m}$ can be derived as follows. Considering the origin of our coordinate system as a phase reference, we note that the difference in the propagation length to a point $\vec{R}_{t x}$ between a wave radiated by an emitter at $\vec{r}_{m}$ and the origin is

$$
\begin{aligned}
\left|\vec{R}_{t x}-\vec{r}_{m}\right| & -\left|\vec{R}_{t x}\right| \\
& =\left|\vec{R}_{t x}\right| \sqrt{1-2 \hat{R}_{t x} \cdot \hat{r}_{m} \frac{\left|\vec{r}_{m}\right|}{\left|\vec{R}_{t x}\right|}+\frac{\left|\vec{r}_{m}\right|^{2}}{\left|\vec{R}_{t x}\right|^{2}}}-\left|\vec{R}_{t x}\right| .
\end{aligned}
$$

Under the special case $\left|\vec{r}_{m}\right| /\left|\vec{R}_{t x}\right| \ll 1$ (which implies that the array aperture is much smaller than the distance to the intended beamforming point), (1) can be Taylor-expanded to yield

$$
\left|\vec{R}_{t x}-\vec{r}_{m}\right|-\left|\vec{R}_{t x}\right|=-\hat{R}_{t x} \cdot \hat{r}_{m}\left|\vec{r}_{m}\right|+\mathcal{O}\left(\frac{\left|\vec{r}_{m}\right|^{2}}{\left|\vec{R}_{t x}\right|}\right) .
$$

The abovementioned propagation length variation manifests itself in the phase propagation term of electromagnetic waves, which, under the substitution of (2), becomes

$$
\begin{aligned}
\exp \left[j k\left(-\hat{R}_{t x} \cdot \hat{r}_{m}\left|\vec{r}_{m}\right|+\mathcal{O}\left(\frac{\left|\vec{r}_{m}\right|^{2}}{\left|\vec{R}_{t x}\right|}\right)\right]\right. & \\
& \approx \exp \left[-j k \hat{R}_{t x} \cdot \hat{r}_{m}\left|\vec{r}_{m}\right|\right],
\end{aligned}
$$

where the above approximation can be made under the far-field condition $\left|\vec{r}_{m}\right|^{2} / \lambda \ll\left|\vec{R}_{t x}\right|$. We note that (3) is the phase difference incurred during transmission of each emitter with respect to the origin. The dual-set of phase differentials can be found for the case of an incident wave from a receive direction, $\hat{R}_{r x}$, in an analogous manner. To keep the notation simpler, we define our new $\hat{R}_{r x}$ to be pointing outward. Combining the two phase terms results in the following phase propagation value:

$$
\exp \left[-j k \hat{R}_{t x} \cdot \hat{r}_{m}\left|\vec{r}_{m}\right|\right] \exp \left[-j k \hat{R}_{r x} \cdot \hat{r}_{m}\left|\vec{r}_{m}\right|\right]
$$

For coherent beamforming to occur, (4) needs to equal 0 for the desired beamforming direction. If a variable unwrapped phase, $e^{-j \delta_{m}}$, is added to the $m$ th emitter, then coherent beamforming will occur for

$$
\delta_{m}=-k\left|\vec{r}_{m}\right|\left(\hat{R}_{t x} \cdot \hat{r}_{m}+\hat{R}_{r x} \cdot \hat{r}_{m}\right) .
$$

We note that, in (5), the required delay for beamforming is given in terms of unwrapped phase $\delta_{m}$. Noting that $k=$ $\omega / c$, the required phase delay is a frequency dependent term. As noted earlier, for wideband signals, a frequencyindependent phase delay will result in data decoherence/ISI. Thus, the delay in (5) is best implemented via a time-delay, which is given by the recast form of (5)

$$
t_{m}=-\frac{\left|\vec{r}_{m}\right|}{c}\left(\hat{R}_{t x} \cdot \hat{r}_{m}+\hat{R}_{r x} \cdot \hat{r}_{m}\right) .
$$

As a simple and familiar example, the case of a 1-D $M$ element array in Fig. 5(b), with branch pitch $d$, is studied. For simplicity, we assume that the array coordinates are given by $\vec{r}_{m}=[0, m d, 0]$. This results in (6) being evaluated as

$$
t_{m}=-\frac{m d}{c}\left(\sin \theta_{t x} \sin \phi_{t x}+\sin \theta_{r x} \sin \phi_{r x}\right)
$$

and, since Fig. 5(b) describes the $x y$ plane, we set $\theta_{r x}=\theta_{t x}=$ $\pi / 2$, which results in (7) reducing to

$$
t_{m}=-\frac{m d}{c}\left(\sin \phi_{t x}+\sin \phi_{r x}\right),
$$

where $t_{m}$ is the delay of the $m$ th branch with respect to the $m=0$ branch at the origin. To ensure that all delays are positive, a common delay to all branches may be added. Thus, (8) can be used to set the internal delay of each emitter to achieve desired reception and transmission angles. Using this expression allows the scalable router to operate as a programmable microwave mirror-the user can set the direction in which the reflections should be sent. While the above example is for a 1-D array, this derivation can be used for 2-D and 3-D routers by using the general form found in (6).

To produce a conventional beam pattern, we must choose a specific direction for either the receive or the transmit. Consider a 1-D 8-branch array with $d=\lambda / 2$ branch spacing, such as that shown in Fig. 5(b), with intended receive direction, $\phi_{r x}=-30^{\circ}$, and intended transmit direction, $\phi_{t x}=60^{\circ}$. The needed branch delays are calculated using (8). Fig. 6 shows the transmit and receive beam patterns for the programed array. The transmit beam pattern shows the relative strength of the radiated beam from the router in any given direction when a signal is incident on the router at $-30^{\circ}$. The receive beam pattern shows how energy incident on the router from any given direction contributes to the transmitted beam at $60^{\circ}$.

\section{B. Peripheral Vision}

While the patterns in Fig. 6 describe the intended behavior of the router, attentive readers may note that, for a given set of branch delays, signals may be received from, and transmitted to, directions other than the intended. We describe this as peripheral vision since the signals incident from outside the directions from which the array is "looking" may be redirected as well. The mathematical justification is apparent from (8) as there are many pairs of $\phi_{r x}$ and $\phi_{t x}$ that satisfy the equation for a given $t_{m}$. While the peripheral vision does not interfere with the primary function of the system, it may be undesirable in certain situations. Fortunately, element position can be used to suppress the router's peripheral vision. 

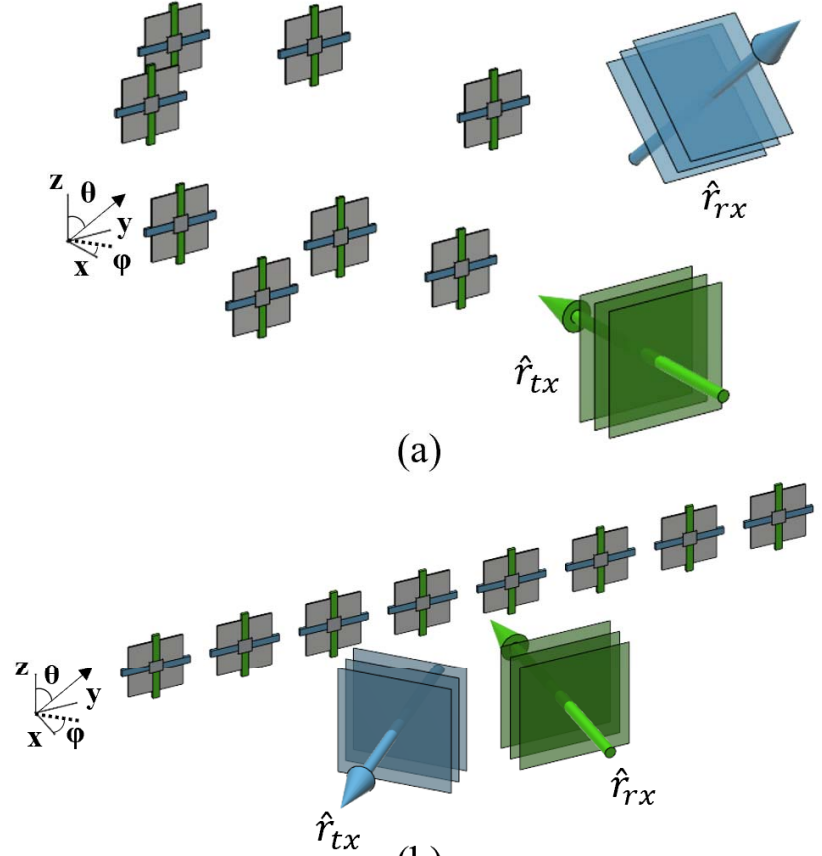

(b)

Fig. 5. (a) Eight-branch router arranged in 3-D space. (b) 2-D eight-branch router with equidistant branch spacing. That spacing is chosen to be $d=\lambda / 2$ for the simulated patterns shown in Fig. 6.

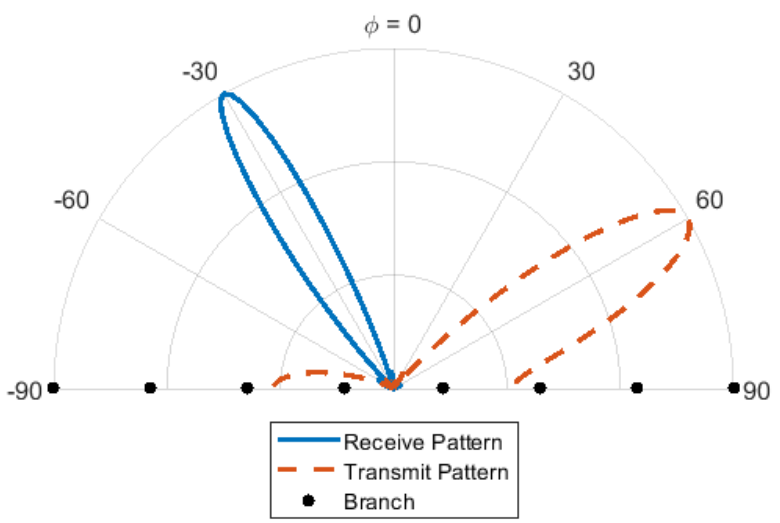

Fig. 6. Normalized linear magnitude pattern plot for 1-D 8-branch linear array with $\lambda / 2$ branch spacing and branch delays programed for an intended $\phi_{r x}=-30^{\circ}$ and $\phi_{t x}=60^{\circ}$.

Router peripheral vision can be quantified for the more general case of a router with steering capability encompassing the entire range of azimuths and elevations. Since the unwanted coherent combination of power is of concern, peripheral vision occurs wherever the carrier signal coherently combines even though the data signal may be incoherent. Focusing on the carrier signal and assuming far-field conditions, the field at a point in space due to a uniformly excited router is proportional to the summation of the propagation phases of each branch

$$
\begin{aligned}
& \left|\vec{E}\left(\hat{R}_{t x}, \hat{R}_{r x}\right)\right| \\
& \propto\left|\sum_{n} \exp \left[-j\left(k \vec{r}_{m} \cdot \hat{R}_{t x}+\delta_{n}+k \vec{r}_{m} \cdot \hat{R}_{r x}\right)\right]\right|,
\end{aligned}
$$

where $\vec{r}_{m}$ denotes the location of the $m$ th branch, and $\hat{R}_{t x}$ and $\hat{R}_{r x}$ denote the instantaneous transmit and receive beam directions, respectively. The $\delta_{m}$ term quantifies the added unwrapped phase by each branch that is used to steer the transmitted beam to a desired $\hat{R}_{\tilde{t x}}$ for a given $\hat{R}_{\tilde{r} \tilde{x}}$ and was defined in (5). In this framework, the problem of minimizing peripheral vision reduces to minimizing (9) for a given set of $\hat{R}_{\tilde{t} x}, \hat{R}_{r \tilde{x}}, \hat{R}_{t x}$, and $\hat{R}_{r x}$ by varying $\vec{r}_{m} .^{3}$

As an example of the effect of branch position, $\vec{r}_{m}$, on peripheral vision, the maximum transmitted power over all $\hat{R}_{t x}$ as a function of $\hat{R}_{r x}$ is shown in Fig. 7 for both a circular and a square router of nine branches. The branches in the routers were programed to transmit at $\hat{R}_{t x}=\left[\phi_{t x}=\right.$ $\left.45^{\circ}, \theta_{t x}=60^{\circ}\right]$ and were intending to receive at $\hat{R}_{r x}=\left[\phi_{r x}=\right.$ $-45^{\circ}, \theta_{r x}=30^{\circ}$ ]. Contours shown in Fig. 7 correspond to the maximum transmitted power in any $\hat{R}_{t x}$ for the given received direction, $\hat{R}_{r x}$, which is described by a point in the $\phi_{r x}-\theta_{r x}$ plane. Note that the router is programed to receive a beam in only a desired $\hat{R}_{\tilde{r x}}$, which corresponds to a single point in the $\phi_{r x}-\theta_{r x}$ plane in Fig. 7. Thus, any contours in Fig. 7 that lie on points in the $\phi_{r x}-\theta_{r x}$ other than the intended $\hat{R}_{\tilde{r x}}$ represent power that is being received from directions other than $\hat{R}_{\tilde{r x}}$ and, subsequently, routed to some unintended transmit direction. The higher the amount of this power (contour level in Fig. 7), the more the peripheral vision is present in the router system. The goal of peripheral vision reduction is to minimize the contours in Fig. 7 so that power is only transmitted when the received beam direction is the intended received beam direction $\hat{R}_{r \tilde{r}}$.

To normalize the comparison between the square and circle routers in Fig. 7, the aperture size of the two arrays is held constant-for a square router with nine branches and $\lambda / 2$ branch pitch, the circular router has $\lambda / 2.25$ branch pitch. Router radiative elements are simulated with a $\cos \theta$ element pattern. As can be seen, the circular router has a better peripheral vision rejection and highlights the importance of branch placement on minimizing peripheral vision. Note that the actual peak of transmitted power does not occur for the intended receive direction, this is due to the effect of the $\cos \theta$ element pattern. More insight into peripheral vision suppression could be obtained by further analysis of (10).

Fig. 8 shows a subset of the above analysis, where, instead of finding the maximum transmitted power over all $\hat{R}_{t x}$ as a function of $\hat{R}_{r x}$, the transmitted power in the $\hat{R}_{\tilde{t x}}$ as a function of $\hat{R}_{r x}$ is shown. This is effectively the amount of undesirable power, other than the intended receive signal $\hat{R}_{\tilde{r x}}$, that is transmitted in the desired transmit direction, $\hat{R}_{\tilde{t} x}$. Once again, to minimize the peripheral vision, we want to minimize the contours in Fig. 8 so that power is only transmitted when

\footnotetext{
${ }^{3}$ In scenarios where there is a maximum undesired power level that can be transmitted due to the peripheral vision, the minimization can be explicitly stated over all space, for a set $\hat{R}_{\tilde{x} \tilde{x}}$ and $\hat{R}_{\tilde{r} \tilde{x}}$, as

$$
\min _{\vec{r}_{m}} \oiint_{S}\left|\vec{E}\left(\hat{R}_{t x}, \hat{R}_{r x}\right)\right|^{2} \mathcal{H}\left(\left|\vec{E}\left(\hat{R}_{t x}, \hat{R}_{r x}\right)\right|^{2}-P_{\max }\right) d S_{r x} d S_{t x}
$$

where the integration over $S$, the unit sphere, captures different $\hat{R}_{t x}$ and $\hat{R}_{r x}$ directions, $\mathcal{H}$ is the Heaviside operator, and $P_{\max }$ is the maximum undesired
} power level. 


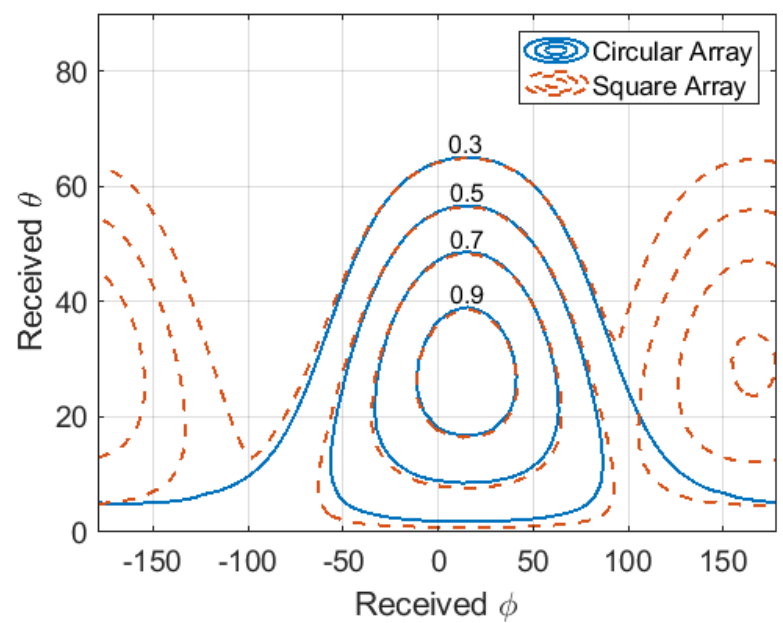

Fig. 7. Contours showing the normalized maximum transmitted power in any $\hat{R}_{t x}$ as a function of $\hat{R}_{r x}$ for both a circular and square routers of nine branches with a fixed aperture size of $\lambda^{2}$.

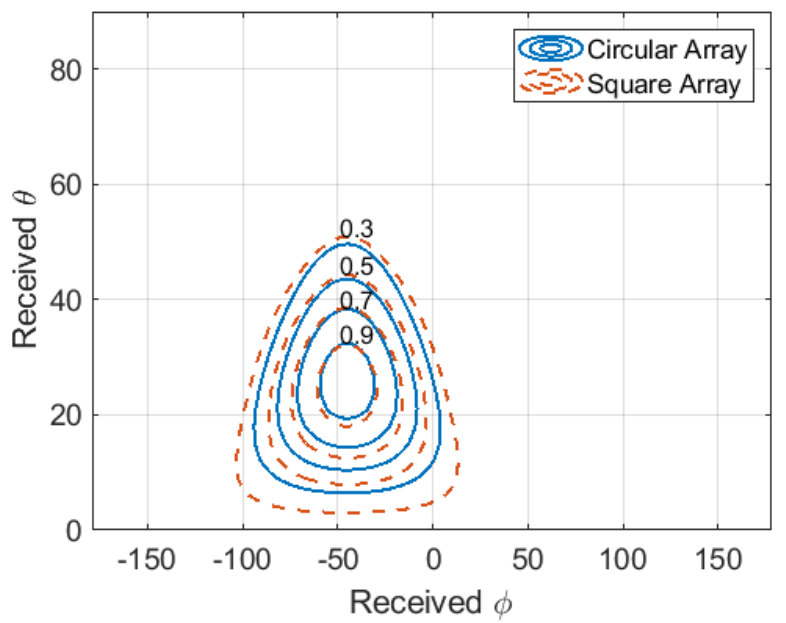

Fig. 8. Contours showing the normalized transmitted power in $\hat{R}_{\tilde{x} \tilde{x}}$ as a function of $\hat{R}_{r x}$ for both circular and square routers of nine branches with a fixed aperture size of $\lambda^{2}$.

the received beam direction is the intended received beam direction $\hat{R}_{\widetilde{r x}}$.

Scalable router peripheral vision is relevant when blocker signals may be present. While out-of-band blockers are filtered by the frequency selectivity of the branch antennas and circuits, large in-band blockers could degrade router performance. The lack of centralization in scalable routers has linearity and blocker tolerance advantages compared with a router constructed from conventional arrays. The greatest amplitude for a blocker signal can occur at the centralized summation node in a conventional array that is avoided in scalable routers. If a blocker signal is high enough power to cause nonlinear effects within individual branch circuitry, the scalable router will exhibit the same signal intermodulation and gain reduction, which occurs in conventional arrays. In addition to frequency and power, the blocker angle of arrival is critical to determining its effect. For the standard, fixed pitch, linear array, a blocker arriving from a direction outside of the intended receive direction will be redirected away from the intended target and will be unlikely to cause any negative effects. For a scalable router with suppressed peripheral vision, a blocker arriving from a direction outside the intended receive direction may not be coherently redirected in any direction. A thorough, probabilistic analysis of blocker suppression and redirection could be performed if router geometry and the likely positions of other relevant transmitters and receivers are known. Unsurprisingly, a high power, in-band blocker arriving at the same orientation as the intended receive direction constitutes a worst case scenario, where the router would rely on branch circuit linearity and the linearity/selectivity of the system that it is routing a signal to for successful operation.

Finally, it should be noted that peripheral vision is only a concern for the router architecture of Fig. 2(c). For example, in a conventional phased array, such as that shown in Fig. 2(b), signal aggregation is done before transmit, and peripheral vision is nonexistent. The hybrid architecture of Fig. 2(d), thus, results in a lower peripheral vision than Fig. 2(c) for the same number of elements. In addition, as mentioned earlier, the peripheral vision described earlier relates to the unwanted coherent combination of the carrier. A peripheral vision where data coherence is maintained is only a subset of the points in the carrier peripheral vision space and is less of an issue for large, spatially distributed arrays operating in wideband networks.

\section{Data Coherence}

The scalable router architecture enables the creation of large aperture arrays (which may be contiguous or physically separated). Data coherence degradation is a natural concern for such systems as ISI and beam squint occur if, within each branch, the phase delay is used instead of TTD [4]. These effects are more pronounced when the wavelength of the highest frequency components of an incident signal's modulation is comparable to array aperture size. This makes large aperture arrays steering high bandwidth beams most susceptible.

For a given beam direction, pure phase control maintains perfect coherence only at a single frequency. In order to preserve beam coherence in a band of frequencies and prevent ISI, an additional degree of freedom must be added. This can be achieved by controlling the slope of each branch's phase response with respect to frequency (i.e., adjusting group delay). Programmable time delay within each branch unlocks system scalability - the primary motivation for the scalable router. While TTD enables high bandwidth arrays, the additional degree of freedom that it affords can alternatively be used to simultaneously and independently control two separate, full power beams. Dual-beam capability is further explained and demonstrated in Section V.

\section{Noise Implications}

To live up to the scalability potential of the distributed router architecture, the highly complex branch circuits must be manufacturable at a low cost and high volumes. Integrated circuits 


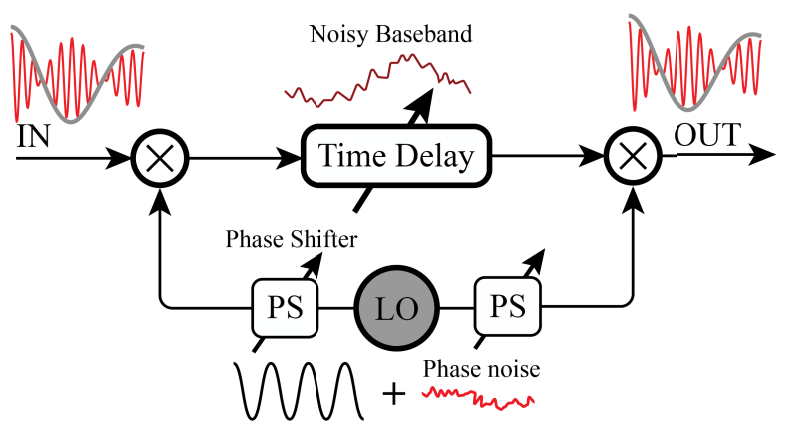

(a)

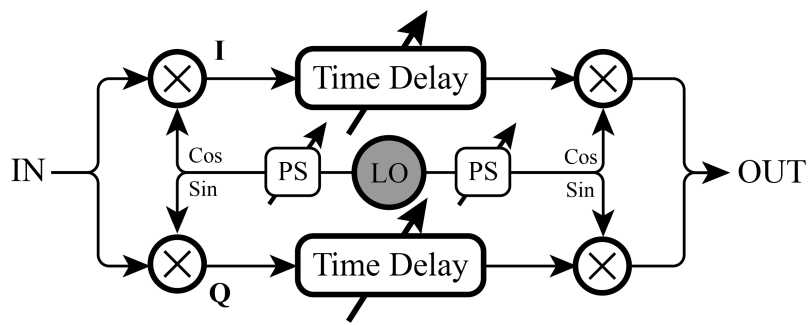

(b)

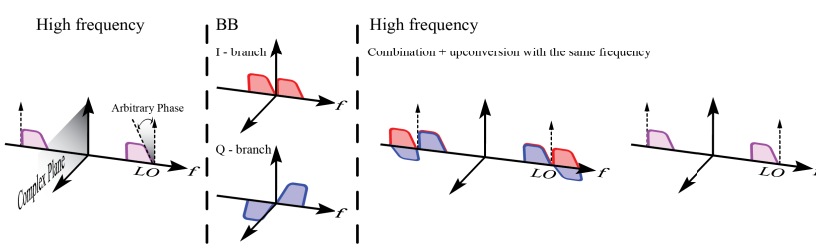

(c)

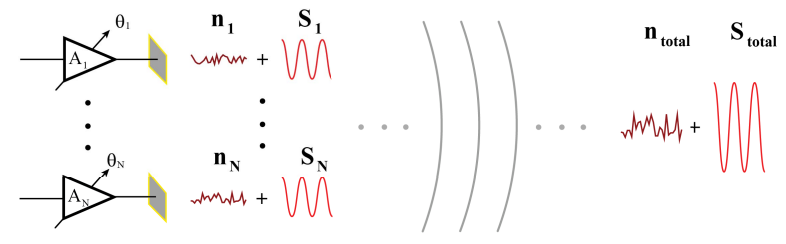

(d)

Fig. 9. (a) Typical branch implementation includes down-conversion and up-conversion of the signal by an LO tone and the application of TTD. (b) To avoid the image issues associated with single sideband mixing, separate I/Q paths may be used. (c) Visual representation of image rejection by the I/Q architecture. (d) Because the scalable router branches are independent and decentralized, the noise added within each branch is uncorrelated.

processes, especially general-purpose complementary metaloxide-semiconductor (CMOS), can deliver this complexity and volume at an attractive cost. An integrated circuit-based scalable router also has the potential to reduce implementation cost and printed circuit board (PCB) complexity compared with a conventional two phased array relays. Without a centralization node, the receive and transmit circuitry can be combined within a single integrated circuit die. An integrated circuit-based implementation also reduces the marginal cost of an additional circuit (such as programmable time delay) needed for a scalable router.
While an integrated circuit implementation has a lot of advantages, it presents a challenge to achieving programmable TTD with a wide range and high resolution at microwave frequencies. Hence, it is preferable to down-convert the received microwave signal and apply TTD at lower frequencies. This architecture is shown in Fig. 9(a). To suppress the signal image, the architecture can incorporate an in-phase/quadrature (I/Q) scheme, as depicted in Fig. 9(b) and (c).

It is noteworthy that the LO within each branch of the scalable router does not have to be phase or frequency locked to the data carrier frequency or other branches. Also, the down-conversion and up-conversion branch architecture serendipitously suppresses the effect of phase noise in the branch LO. To understand this effect, consider an input to the I/Q branch as

$$
X(t)=I(t) \cos \left(2 \pi f_{0} t\right)+Q(t) \sin \left(2 \pi f_{0} t\right),
$$

where $f_{0}$ is the incoming wave frequency. After downconversion, we would have

$$
\begin{aligned}
X_{I}= & \frac{I(t)}{2} \cos \left[2 \pi\left(f_{\mathrm{LO}}-f_{0}\right) t+\phi_{\mathrm{LO}}(t)+\phi_{\mathrm{PR}}\right] \\
& -\frac{Q(t)}{2} \sin \left[2 \pi\left(f_{\mathrm{LO}}-f_{0}\right) t+\phi_{\mathrm{LO}}(t)+\phi_{\mathrm{PR}}\right] \\
X_{Q}= & \frac{I(t)}{2} \sin \left[2 \pi\left(f_{\mathrm{LO}}-f_{0}\right) t+\phi_{\mathrm{LO}}(t)+\phi_{\mathrm{PR}}\right] \\
& +\frac{Q(t)}{2} \cos \left[2 \pi\left(f_{\mathrm{LO}}-f_{0}\right) t+\phi_{\mathrm{LO}}(t)+\phi_{\mathrm{PR}}\right],
\end{aligned}
$$

where $f_{\mathrm{LO}}$ is the $\mathrm{LO}$ frequency, $\phi_{\mathrm{LO}}(t)$ is the associated phase noise, and $\phi_{\mathrm{PR}}$ is the applied phase shift. ${ }^{4}$ The output signal after applying TTD and up-converting is

$$
\begin{aligned}
& X_{\mathrm{out}}= \frac{I(t-\tau)}{2} \cos \left[2 \pi f_{0}(t-\tau)-\phi_{\mathrm{PR}}+\phi_{\mathrm{LO}}(t-\tau)-\phi_{\mathrm{LO}}(t)\right] \\
&+\frac{Q(t-\tau)}{2} \sin \left[2 \pi f_{0}(t-\tau)-\phi_{\mathrm{PR}}\right. \\
&\left.\quad+\phi_{\mathrm{LO}}(t-\tau)-\phi_{\mathrm{LO}}(t)\right] .
\end{aligned}
$$

As shows in (18), the transmitted frequency is exactly at $f_{0}$. This result is independent of each branch LO frequency $\left(f_{\mathrm{LO}}\right)$. Since the delay, $(\tau)$, is on the order of picoseconds to few nanoseconds, the resultant additional phase noise, due to the term $\phi_{\mathrm{LO}}(t-\tau)-\phi_{\mathrm{LO}}(t)$, is negligible up to offset frequencies in the gigahertz range. This near-complete phase noise cancellation bolsters the scalable routers potential for distributed operation as inexpensive reference oscillators with relaxed stability [such as cheap crystal oscillators or on-chip freerunning voltage-controlled oscillator (VCO)] may be used. A measurement of the implemented branch circuit shown in Fig. 10 demonstrates this phenomenon clearly. A tone at $25.01 \mathrm{GHz}$ is sent through a branch of the IC (whose details will be discussed in Section IV with a free-running VCO around $24.98 \mathrm{GHz}$. The image at $24.95 \mathrm{GHz}$ exhibits twice the phase noise of the $\mathrm{VCO}$, while the retransmitted tone has a clean spectrum without any of the VCO phase noise.

\footnotetext{
${ }^{4}$ It is implicitly assumed in (12) that the phase noises of the I and Q are correlated. This is generally a valid assumption if they are generated within the same oscillator core.
} 


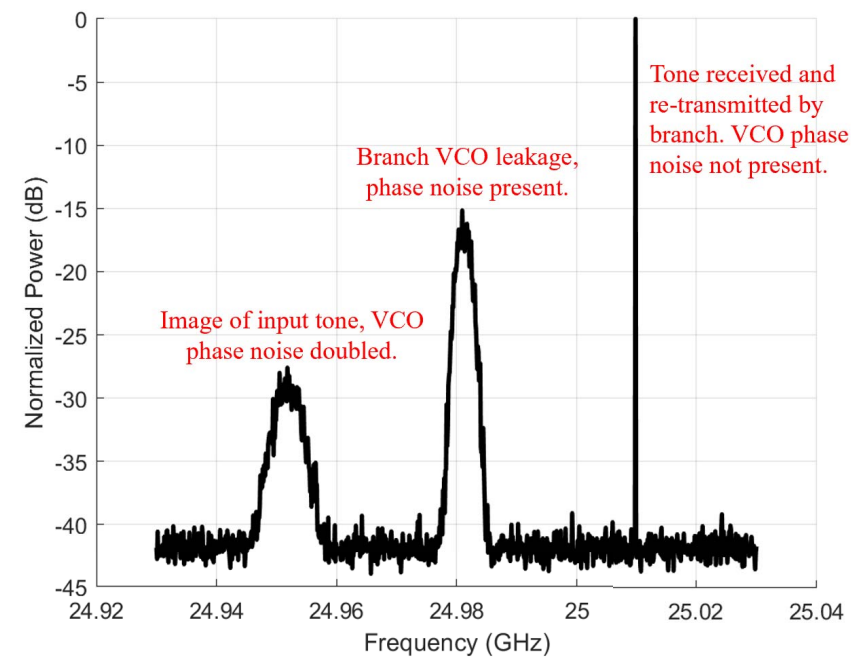

Fig. 10. Radiative measurement of the output spectrum of a branch with free-running VCO and excited by an external source. The branch is digitally configured to maximize VCO leakage through an up-conversion mixer for better observation of phase noise cancellation.

In addition to phase noise cancellation, the scalable router architecture also provides mitigation of added amplitude noise within each branch. Due to the transceiver branches being fully separate and the absence of any kind of physical summation node within the system, the added noises (antenna noise temperature, noise added by amplifiers, and so on) are uncorrelated. This lack of correlation due to decentralization, as shown in Fig. 9(d), results in higher SNR at the target compared with a relay constructed from traditional, centralized arrays. This noise reduction can be leveraged to trade component-level noise performance for other system benefits, for example, reduction of capacitance in a switched capacitor filter to increase bandwidth and reduce on-chip area at the cost of added uncorrelated noise. Decentralization can also help reduce the effect of delay and phase shift quantization noise or setting errors. Uncorrelated stochastic variations or deterministic errors in individual branches are incoherently combined in the transmitted beam of the router, blunting their effect.

\section{E. Branch Isolation and Self-Interference}

Scalable routers are not immune to the self-interference issues that plague many simultaneous transmission/reception (full-duplex) systems. In particular, parasitic feedback from the transmitter output back to the input of the receive chain interferes with system function even if it is far below the levels necessary to cause oscillation. Consider the simplified, frequency-independent branch with forward path gain $\alpha$ and parasitic feedback $\beta$ shown in Fig. 11. The delay element within the branch is an ideal constant amplitude phase rotator. The branch's closed-loop transfer function phase and normalized amplitude are plotted versus phase rotator setting for several open-loop gains $(\alpha * \beta)$ in Fig. 11. The parasitic feedback introduces nonidealities to the previously ideal phase rotator. An open-loop gain of $-20 \mathrm{~dB}$ produces nearly ideal

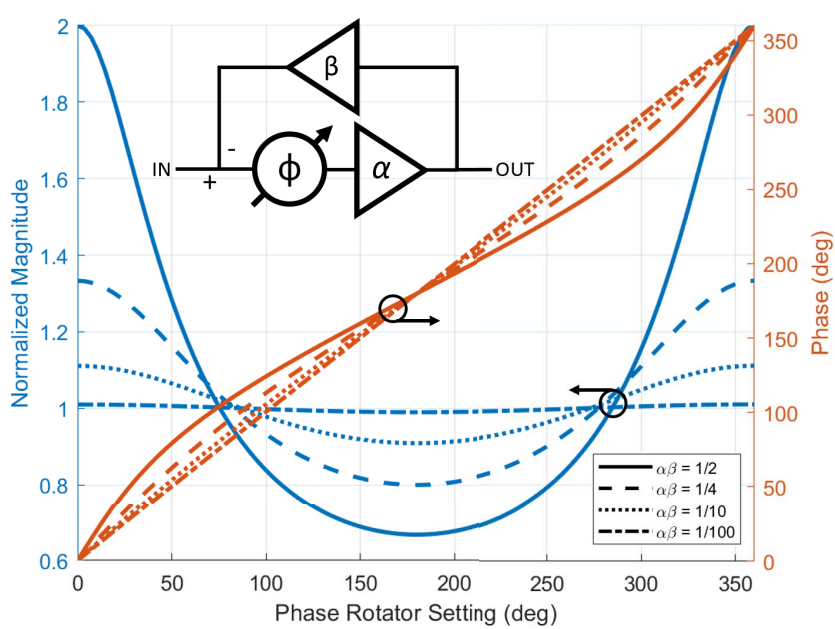

Fig. 11. Simplified branch transfer function phase and amplitude as ideal, unity gain phase rotation occur under the presence of parasitic feedback.

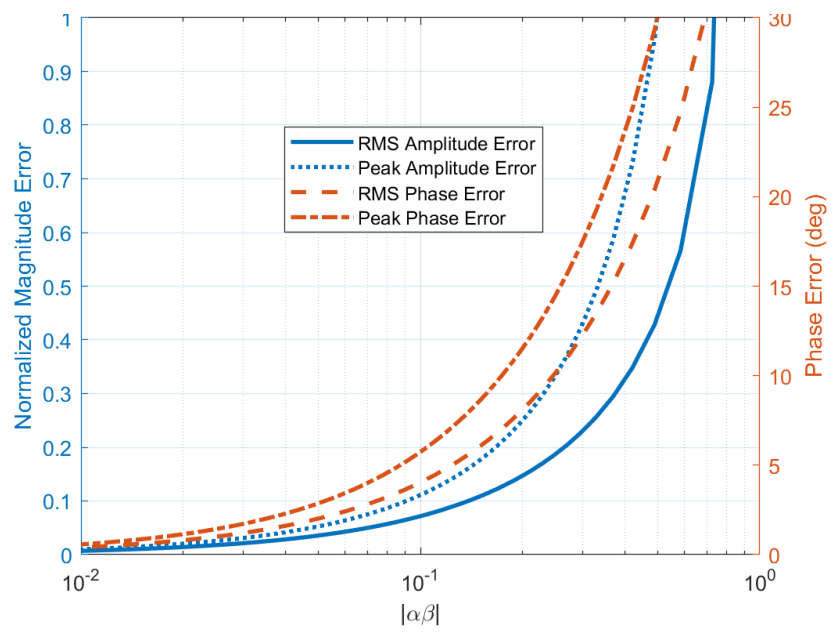

Fig. 12. Peak and rms transfer function phase and amplitude error versus the open-loop gain of the simplified branch model.

behavior but nonideality quickly emerges as this gain rises. The dependence of these nonidealities on open-loop gain can be observed in Fig. 12. Fig. 12 shows the amplitude and phase error (deviation from the ideal) as the open-loop gain is changed. Even at an open-loop gain of 0.1 , the peak phase error exceeds $5^{\circ}$. These nonidealities limit the achievable branch forward path gain, as a gain of $30 \mathrm{~dB}$ would require isolation of close to $50 \mathrm{~dB}$ for the peak amplitude and phase variations to be rendered unnoticeable. Polarization isolation, isolating radiators on opposite sides of a ground plane, or active feedback cancellation techniques can reduce parasitic feedback to acceptable levels. Provided that the branches are implemented by integrated circuits, the additional complexity of active cancellation circuits comes at a low marginal cost. While coupling between adjacent branches may also be a concern, the isolation within a branch is likely to be worse than the isolation between branches of even a dense ( $0.5 \lambda$ pitch) array. 


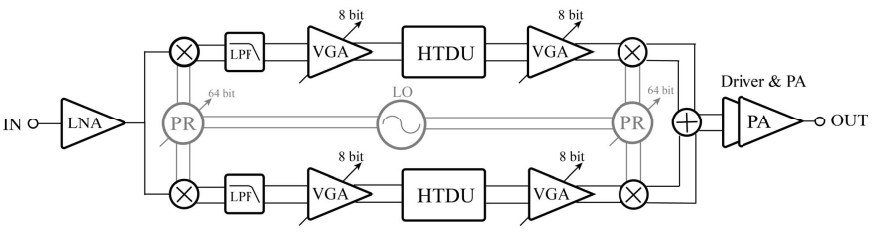

(a)
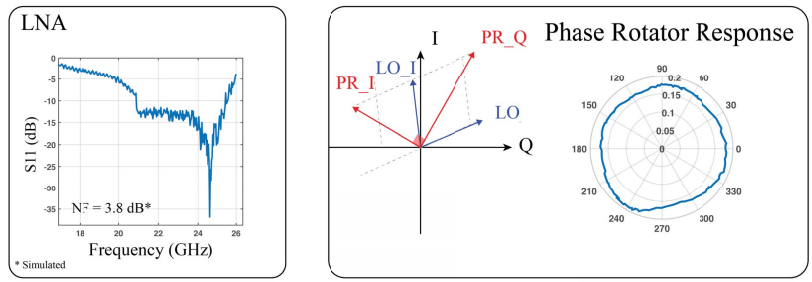

(b)
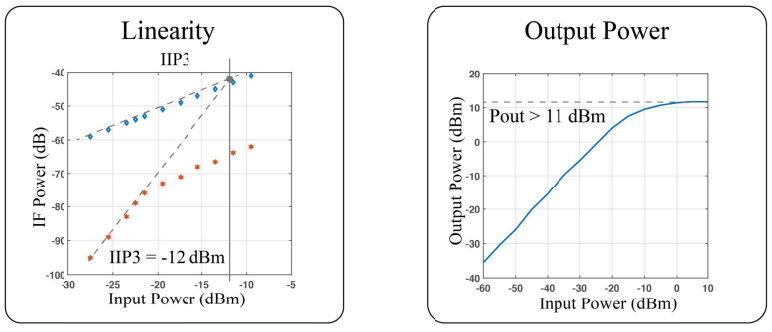

(c)

Fig. 13. (a) Branch integrated circuit architecture. (b) LNA matching and vector sum phase rotator performance. (c) System linearity and PA output power.

\section{BRANCH CiRCUIT}

The presented router branches are implemented fully on a monolithic CMOS IC with programmable gain and TTD. CMOS ICs are well suited for a scalable router as they not only compactly combine the many digital and analog functions needed for array operation but also offer low cost at high volume. Favorable cost scaling is crucial for the router architecture as the cost is as formidable an obstacle as any circuitlevel performance specification for widespread adoption of arrays of hundreds or thousands of elements. The primary function of the branch circuit is to provide amplification and programmable TTD to its received signal. The presented branch circuitry uses a baseband hybrid analog/digital time delay unit (HTDU). The branch architecture is shown in Fig. 13(a). A branch begins with a low-noise amplifier (LNA) followed by an I/Q down-conversion mixer. The I/Q mixer's LO is generated on-chip. The LO signals pass through vector sum phase rotators that provide $360^{\circ}$ phase control. Variable gain amplifiers condition the baseband I/Q signals before they are sent to the HTDU. After the delay unit, the I/Q signals are up-converted using similar LO signals that undergo independently controlled phase rotation. The up-converted $\mathrm{I} / \mathrm{Q}$ signals are recombined at $\mathrm{RF}$ in a vector summer and transmitted by the driver and the PA. Fig. 13(b) and (c) shows LNA input matching, receiver chain IIP3, system gain, output power, and compression curves. The die micrograph of the branch IC is shown in Fig 14.

Time delay with fine resolution control and broad variation range is critical to the decentralized router's scalability.

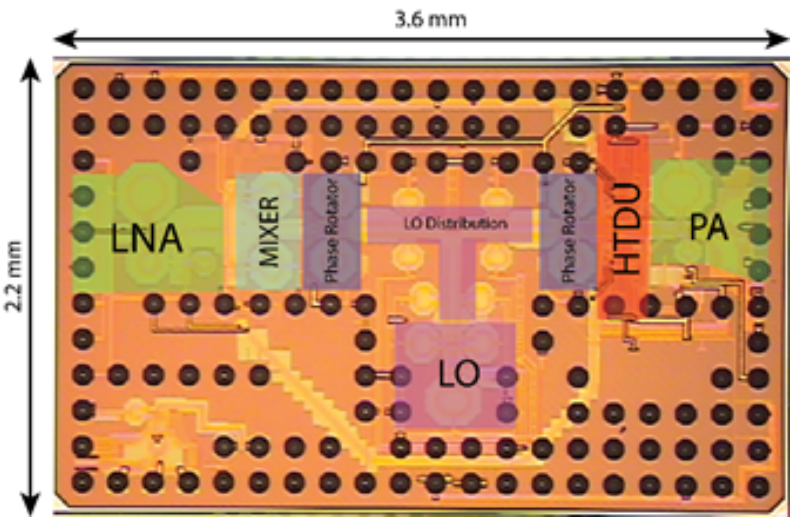

Fig. 14. Die photo of branch integrated circuit implemented in standard 65-nm CMOS process.

Large-scale timed arrays are challenging to implement because many existing integrated delays are power-hungry, areainefficient, or lacking sufficient range and resolution. Non-IC solutions, such as switchable transmission lines or optical delays, are physically and economically impractical for arrays with hundreds or thousands of elements.

The scalable router HTDU performance requirements are determined by the physical array aperture size and bandwidth requirements of the intended application. For instance, in a $1-\mathrm{m}$ aperture span array with $500-\mathrm{MHz}$ baseband bandwidth, two elements on opposite sides of the array may experience time offset of up to $3 \mathrm{~ns}$ while requiring delay adjustment resolution of $<20 \mathrm{ps}$ for temporal alignment within $1 \%$ of the maximum data frequency content. The 3-ns delay range requirement is larger than existing analog delay solutions [15]-[17], while the $<20$-ps resolution is challenging in purely digital solutions due to unrealistic digital clock requirements [18]. This work uses a hybrid analog/digital switched capacitor delay unit, which fulfills both requirements. A hybrid ana$\log$ /digital delay unit concept intended for large array applications was recently presented in [19]. Any switched capacitor circuit can be considered as a time delay. The signal is sampled onto the capacitor by a clock edge delivered to the input switch and accessed later by a subsequent clock edge. By controlling the delay between the sample and access clock edges, the delay of the circuit can be changed. To provide a sufficient sample rate for the input signal bandwidth, multiple switched capacitors are placed in parallel in an N-path configuration. The input and output switches are controlled by two separate nonoverlapping clock (NOC) generators. The time delay is controlled by the relative phase of the two NOCs.

The HTDU (schematic shown in Fig. 15) uses eight switched capacitors in parallel and has fine, medium, and coarse controls of the relative phase of the NOCs. The fine and medium controls change the phase of the clock driving the output NOC. The fine control uses a DAC to change the bias of a chain of current starved inverters carrying the clock signal. The medium control adds or removes inverters to/from the output clock signal path. The coarse control changes the location of the pulse in the output NOC. The delay element measurements in Fig. 16 show 5 ns of range with a minimum 


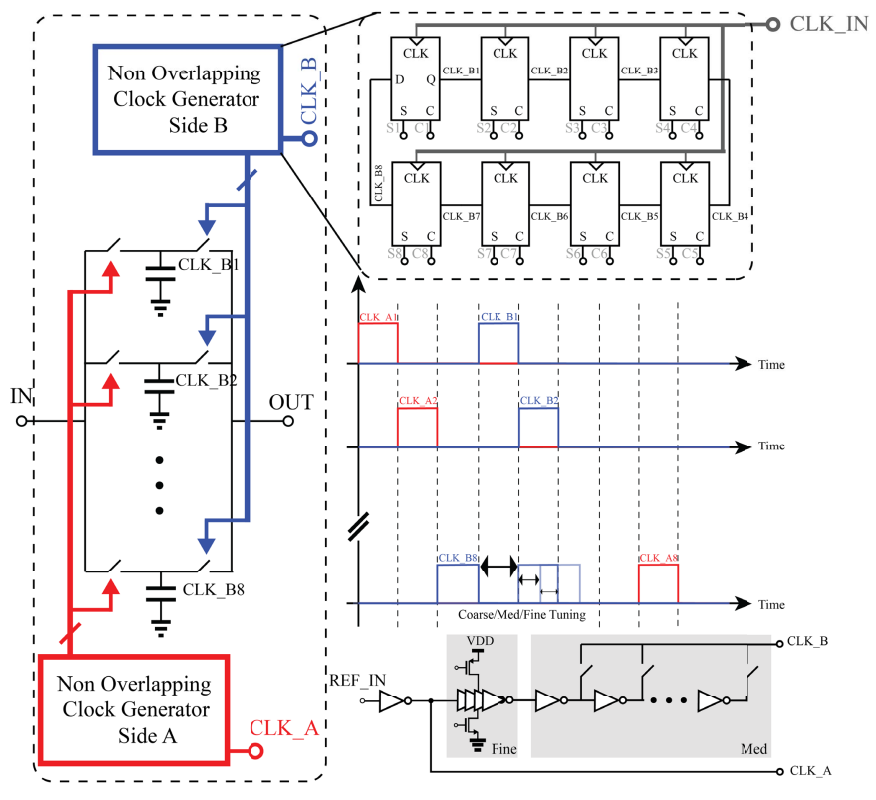

Fig. 15. HTDU. Coarse delay is controlled by the initial set/reset state of the NOCs. Medium delay adds or removes inverters, and fine delay changes a voltage-controlled delay line.

step size of $5 \mathrm{ps}$ for the fine step. The minimum step is defined as the smallest step that the nonlinear fine delay setting control could be linearized to while still utilizing its full range. The measured delays are determined by fitting a line to the measured phase response and taking its slope (group delay). Digital code " 0 " for the coarse delay represents a state when the clock pulse for reading from the delay capacitors overlaps the writing clock pulse as such digital code " 1 " is used for normalization instead. Simulation/analysis curves for coarse, medium, and fine delays are based on extracted delay cell simulations from which the delay range is calculated rather than full-system top-level simulations. The coarse delay step and range can be increased or decreased by adjusting the NOC clock frequency, with the maximum achievable $10 \mathrm{~ns}$ of delay demonstrated in the radiative measurements. In our implementation, the input clock can operate in any frequency from 650 to $4 \mathrm{GHz}$. The clock frequency of $650 \mathrm{MHz}$ (corresponding to a 10-ns delay) is the minimum value for which all the delays in the range can be generated without a gap.

\section{System Measurements}

\section{A. Scalable Router Prototype and Demonstration}

A radiative, four-branch, receive and transmit capable, scalable router prototype was built. Each branch IC is mounted on a PCB with orthogonally polarized patch antennas. The scalable router is formed using a number of these branch PCBs arranged in the desired spatial configuration. The branch circuit board, the simulated patch antenna S-parameters (input matching and isolation), and the radiation pattern are presented in Fig. 17. The simulated isolation between the antennas is $\sim 50 \mathrm{~dB}$ - high enough to not induce significant feedback effects.

The scalable router architecture's potential in large-scale array applications where there is no shared timing reference between branches is demonstrated in the test setup depicted in Fig. 18. The branches are placed in two pairs separated by $1.5 \mathrm{~m}$. A transmit/receive horn antenna pair is placed $1 \mathrm{~m}$ from the leading branch pair. This transmit and receive horn antenna pair is used to excite the router and measure its reradiated beam. The total round-trip path length difference between the front pair and back pair is approximately $3 \mathrm{~m}$, which corresponds to 10-ns delay. The branch circuits share no timing information and use internal free-running VCOs to provide the LO signal for the circuit.

To illustrate the routers functionality and the importance of TTD for the scalable router, two digital configurations of the router were measured. The first configuration steers the routed beam using only the phase rotators. This matches the elements' phases at only a single frequency point. The 10-ns delay mismatch between the branch pairs causes a difference in group delay (slope of the phase response) and prevents a coherent combination of the branches' signals outside of a narrow bandwidth. The second configuration uses TTD in addition to the phase rotators to match the branch phase responses over a frequency band. By connecting a vector network analyzer (VNA) to the transmit and receive antennas of the setup, the response of each branch can be measured individually. Fig. 19 shows the measured phase response and group delay of each branch with and without TTD correction. The measurements with TTD clearly illustrate the matched phase and group delay for all four branches, demonstrating the TTD adjustment capability of the branch circuit. The peaks in group delay are caused by LO leakage of each branch circuit.

The coherence restored by the branch circuit TTD is critical for the transmission of data by large arrays. Without this correction, ISI degrades the rerouted data. The same test setup and branch configurations discussed earlier were excited by a 24.96-GHz signal modulated with BPSK and 16- and 64-QAM data streams at $45 \mathrm{MS} / \mathrm{s}$. The rerouted signals were measured and demodulated. No equalization was used in the measurement setup. The BPSK eye diagram and 16- and 64-QAM constellations and results of the demodulation are shown in Fig. 20. The images on the left-hand side correspond to phase-only steering, while the images on the right-hand show the results with combined phase and time delay steering. The addition of TTD noticeably improves the BPSK eye diagram and improves its EVM from $11.4 \%$ to $5.2 \%$, while the 16-QAM EVM is improved from $8.6 \%$ to $4.4 \%$. The $64-$ QAM constellation is changed from nearly unrecognizable with phase-only steering to an EVM of $4 \%$ with phase and time delay steering. ${ }^{5}$

\footnotetext{
${ }^{5}$ The $4 \%$ EVM may be slightly optimistic as several points at the edges of the constellation may be misidentified as the incorrect symbol. Despite this, the improvement provided by TTD is undeniable.
} 


\section{Coarse}
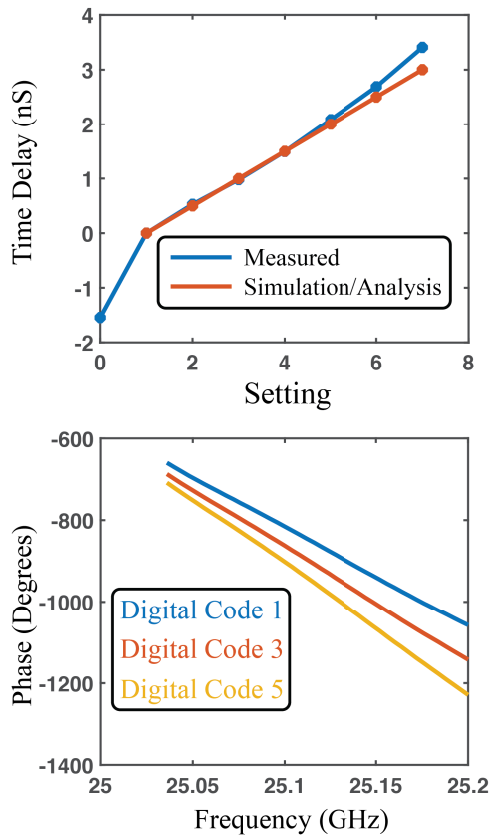

Medium
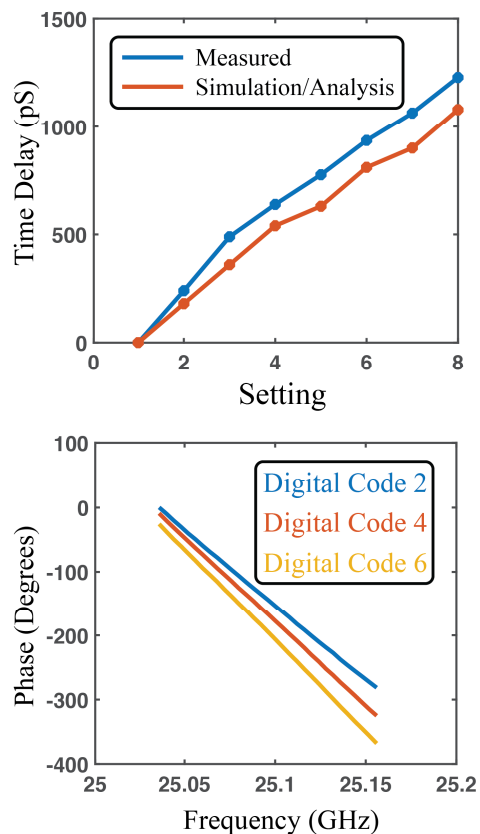

Fine
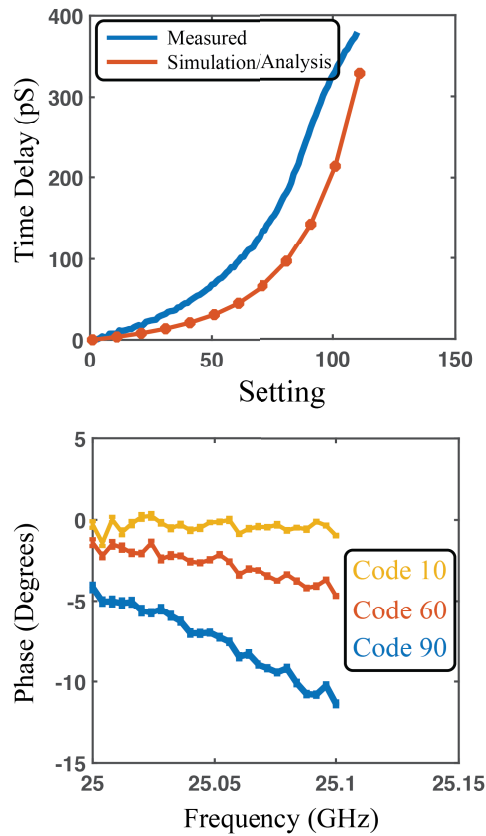

Fig. 16. Coarse, medium, and fine TTDs and measured phase responses for span of coarse, medium, and fine delay settings. All measurements are referenced to the lowest delay setting.

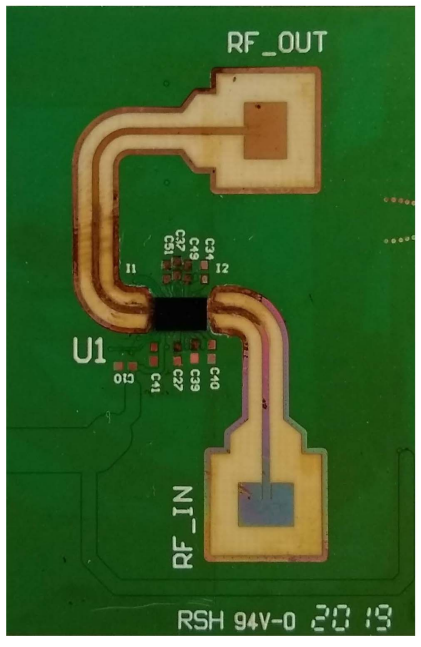

(a)

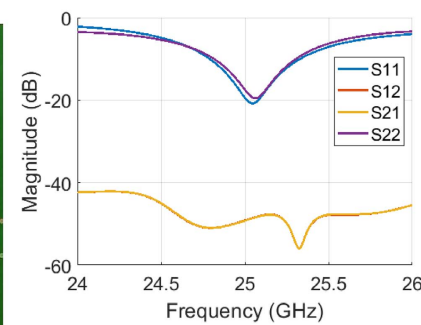

(b)

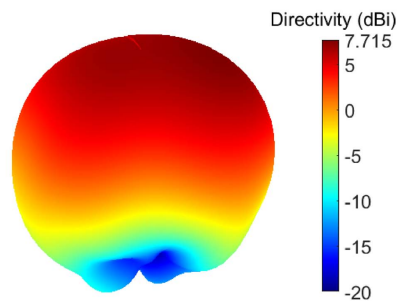

(c)

Fig. 17. (a) Fabricated branch transceiver PCB for radiative measurements of the designed branch integrated circuit. The TX and RX antennas are orthogonally polarized. (b) Simulated S-Parameters of the PCB. (c) Simulated radiation pattern of the designed patch antenna.

While $45 \mathrm{MS} / \mathrm{s}$ is sufficient to observe the importance of TTD correction, it is even more critical at higher modulation rates. The bandwidth of the presented system is limited by unintentional down-tuning of the branch LOs in the branch integrated circuit.
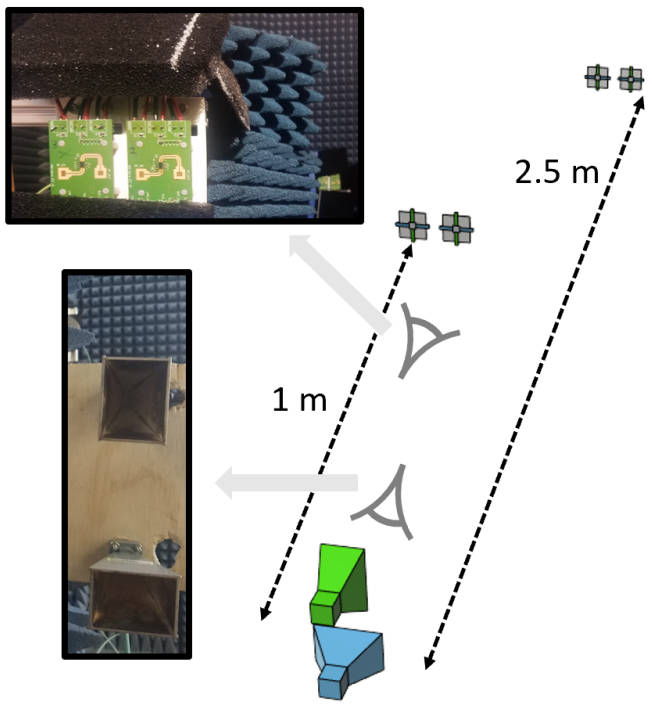

Fig. 18. Radiative scalable router test setup. Two pairs of branch circuits are excited by a horn antenna, and their reradiation is measured by the other horn antenna. The branches are not colocated and do not share a timing/phase reference.

\section{B. Dual-Beam Demonstration}

The TTD capability within each branch can also be used to independently steer beams at two different frequencies. A phased array forms a beam when the phases of the signals radiated by each element in the array match in the desired direction, creating constructive interference. A dual-beam 

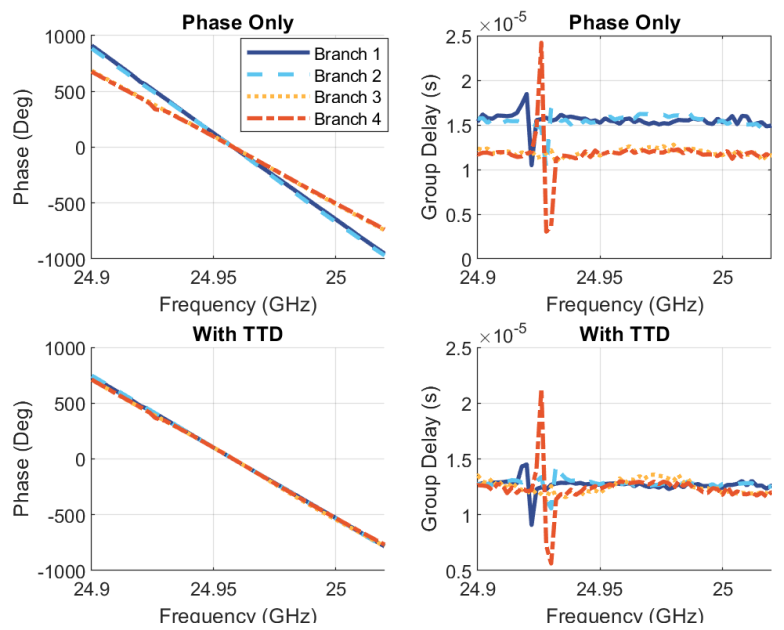

Fig. 19. Radiatively measured branch phase response and group delays with and without TTD correction.

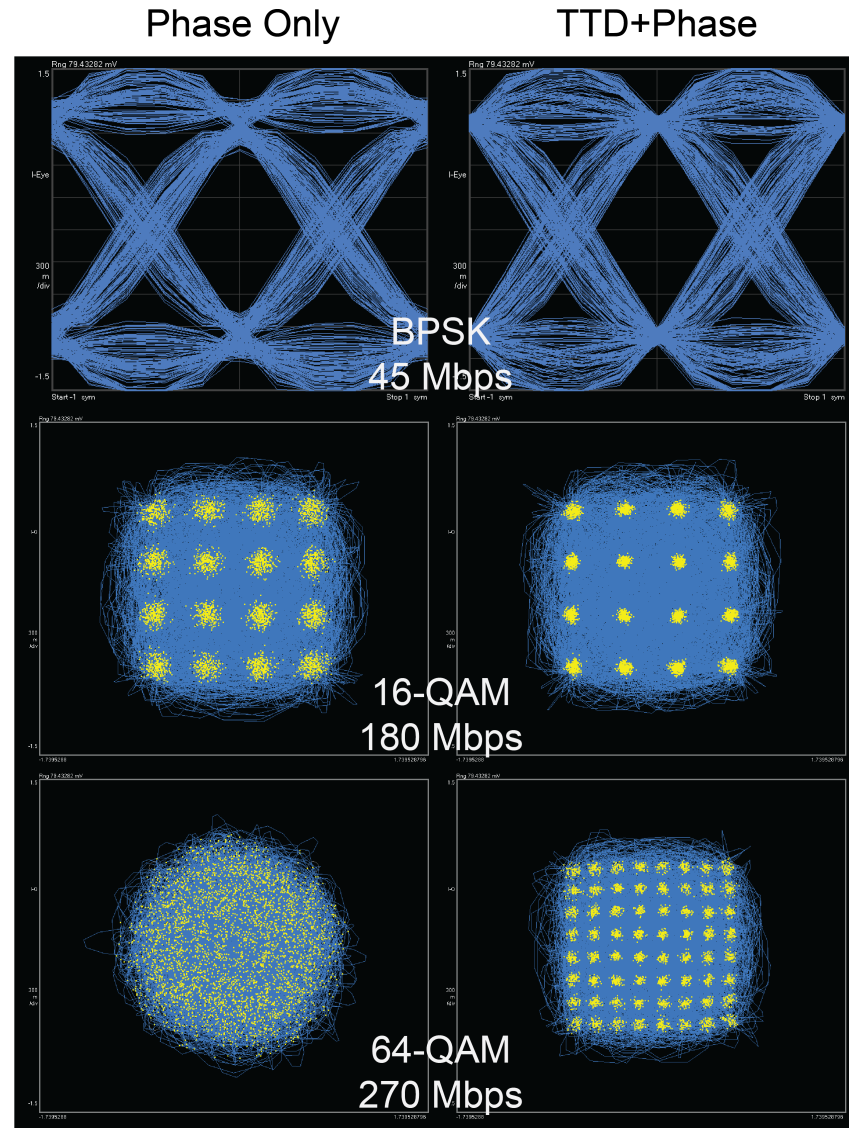

Fig. 20. Received eye diagrams/constellations for BPSK, 16-QAM, and 64QAM at $45 \mathrm{MS} / \mathrm{s}$ with beamforming achieved via phase-only steering (left) or TTD and phase steering (right).

array requires this constructive interference to occur in two desired and potentially arbitrary directions at two different frequencies. Programmable TTD allows for the phase response slope (group delay) of an element to be changed, while a programmable phase rotator changes the phase response offset or intercept. In the previous measurement, we used these two degrees of freedom to match the offset and slope of multiple

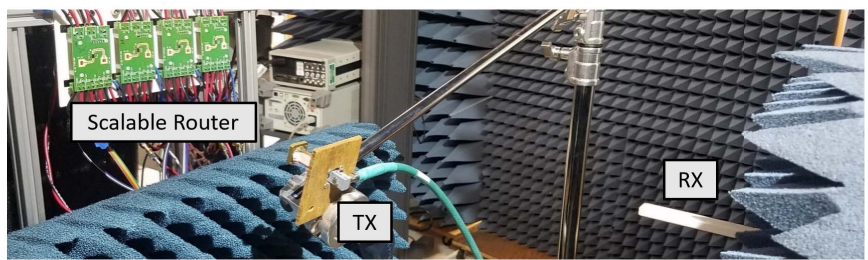

Fig. 21. Dual-beam test setup. TX and RX antennas connect to VNA. $\mathrm{RX}$ antenna is mounted on a linear scanner.
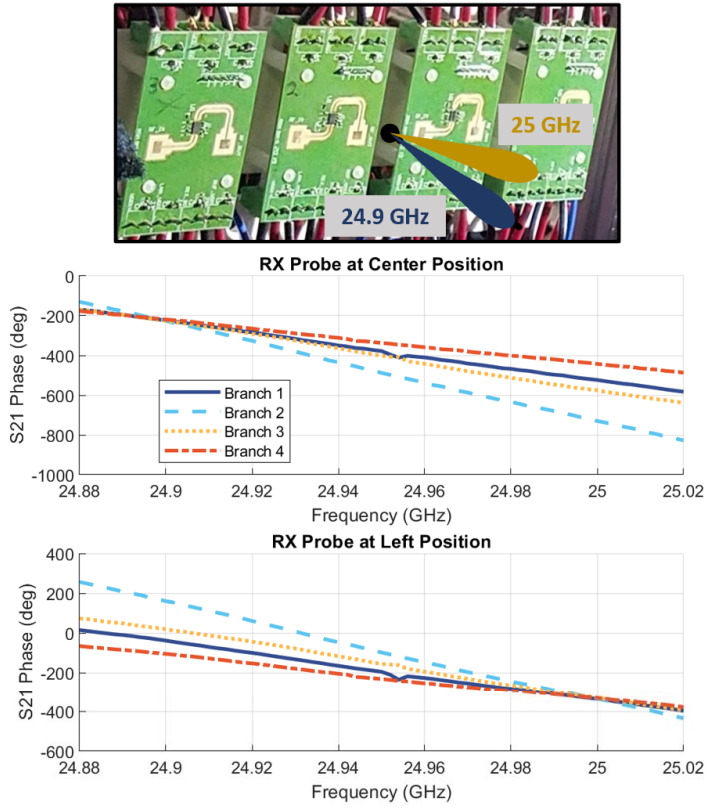

Fig. 22. Branch phase responses measured at the center and left RX probe positions for the router digital configuration which steers $24.9 \mathrm{GHz}$ to the center and $25 \mathrm{GHz}$ to the left.

branches over a band of frequencies to prevent ISI, but they can also be used to match the phase response of the branches at one frequency in one direction and another frequency in another direction. This, in effect, creates two independently controlled full power beams from the array.

To demonstrate the dual-beam capability, the test setup shown in Fig. 21 was built. A four-branch scalable router is radiatively excited by an antenna connected to one port of a VNA, and the rerouted signal is measured by an antenna that is connected to the other port of the VNA and mounted on a linear scanning platform. The transmit antenna is $25 \mathrm{~cm}$ from the center of the scalable router, slightly offset beneath it. The receive antenna (mounted on the linear scanner) is $55 \mathrm{~cm}$ from the center of the router. The dual-beam capability of the scalable router is demonstrated by maintaining a broadside beam at $24.9 \mathrm{GHz}$ while simultaneously steering a beam at $25 \mathrm{GHz}$ to three different locations (center, left, and right). The steering positions are separated by $5 \mathrm{~cm}$ (close to $5^{\circ}$ off the broadside direction) and are chosen in order to stay within the grating lobes caused by the transmit antenna pitch of $5.5 \mathrm{~cm}$.

Fig. 22 shows the measured $S_{21}$ phase for all branches for the configuration where the $24.9-\mathrm{GHz}$ beam is steered 

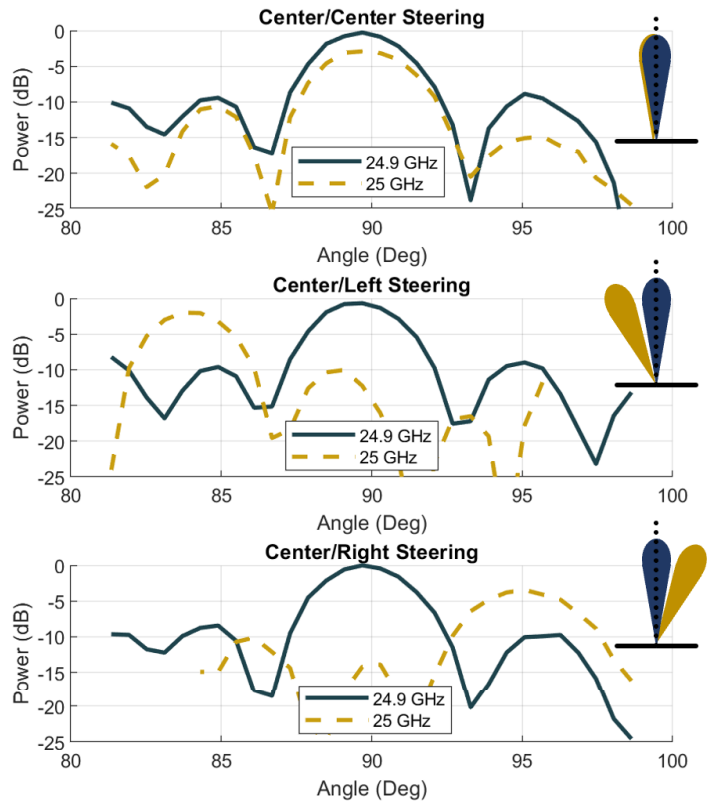

Fig. 23. Measured patterns at 24.9 and $25 \mathrm{GHz}$ for three different steering configurations demonstrate simultaneous, independent controls of two receive/transmit beam pairs. The powers are normalized to the same global maximum occurring on the $24.9-\mathrm{GHz}$ beam during center/center steering.

broadside and the $25-\mathrm{GHz}$ beam is steered left. The phase is measured at two locations: broadside and the position corresponding to the left-steered beam. The constructive interference responsible for beamforming is evident by the matched phase for all elements at $24.9 \mathrm{GHz}$ for the center probe position and at $25 \mathrm{GHz}$ for the left probe position. Fig. 23 shows successful steering of the beam at $25 \mathrm{GHz}$, while the broadside beam at $24.9 \mathrm{GHz}$ stays constant. The leftand right-steered traces have been trimmed to prevent grating lobes (at $\sim 15^{\circ}$ away) from appearing on the opposite side. Static reflections caused by other objects near the setup were measured separately and subtracted from the presented results.

In order to arbitrarily steer beams at two frequencies, the branch circuits must be able to change their relative phase to any value from $0^{\circ}$ to $360^{\circ}$. Since our system accomplishes this relative change using time delay, the period of the minimum frequency separation of two frequencies that can be fully independently steered is the maximum achievable time delay. Thus, $100-\mathrm{MHz}$ separation is the smallest achievable for $10 \mathrm{~ns}$ of delay control.

Given the obvious advantages of a single array serving multiple users, multibeam microwave communication systems have been an active area of research for several decades. An overview of state-of-the-art multibeam approaches as of 2017 can be found in [20]. When considering the wide variety of approaches and subsequent tradeoffs, a direct comparison between the systems is not always apt. Dividing a larger array into independent subarrays is a common technique usable with no additional hardware, but it divides power and aperture between the beams. The following array hardware paradigms (as well as the scalable router dual-beam capability)
TABLE II

PERFormanCE COMPARISON

\begin{tabular}{|c||c|c|c|}
\hline & This work & {$[18]$} & {$[19]$} \\
\hline \hline Application & $\begin{array}{c}\text { Decentralized } \\
\text { TTD Router }\end{array}$ & $\begin{array}{c}\text { Digital TTD } \\
\text { Receiver }\end{array}$ & $\begin{array}{c}\text { Hybrid TTD } \\
\text { Receiver }\end{array}$ \\
\hline Technique & Hybrid & Digital & Hybrid \\
\hline Delay Range [ps] & $0-10000^{\mathrm{w}}$ & $0-7500$ & $0-15000$ \\
\hline Delay Step [ps] & 5 & 250 & 5 \\
\hline Analog Gain [dB] & $26^{\mathrm{x}}$ & - & - \\
\hline Delay Area [mm $\left.{ }^{2}\right]$ & $0.3 / 0.5^{\mathrm{y}}$ & 0.28 & 0.57 \\
\hline Power [mW] & $8 / 70^{\mathrm{y}}$ & $16 / 196^{\mathrm{z}}$ & 47 \\
\hline Technology & $65 \mathrm{~nm}$ CMOS & 40 nm CMOS & 65 nm CMOS \\
\hline Demonstration & Fully & $\begin{array}{c}\text { Connectorized, } \\
\text { Computed } \\
\text { Padiative }\end{array}$ & $\begin{array}{c}\text { Connectorized, } \\
\text { Computed } \\
\text { Pattern }\end{array}$ \\
\hline
\end{tabular}

w. Adjustable with reference frequency.

$\mathrm{x}$. Includes both RF and baseband gain.

y. Baseband delay only/Includes up and down conversion mixers and LO z. ADC power/DBF power

achieve multiple beams without sacrificing power in the beams.

A well-established family of multibeam systems is multiport passive (or semiactive) networks used to create a predetermined set of beam patterns. These arrays can be transmit or receive, have been fully integrated [21], and can create a multitude of beams at the cost of design complexity. However, these arrays are not electronically steerable and require separate input drivers to achieve their multibeam capability.

Another common family of multibeam arrays is the digital arrays, which process the same received signals in several parallel channels [18], [22]. While these systems can create as many steerable beams as processing power and time are available, the topology has only been shown for receive arrays, not transmit arrays. Furthermore, there can be dynamic range limitations due to the analog-to-digital conversion process.

The scalable router dual-beam capability (enabled by programmable time delay) differs from the previously described paradigms as it derives its two beams by "frequency multiplexing" the array. By tuning the phase response of the elements at two frequencies, two independent beams are created. It should be noted that transmit dual-beam capability is not unique to the scalable router architecture. While programmable TTD means that the router is naturally suited to the task, any transmit array with independent phase and group delay control within each element could achieve it. Because this control is established through analog circuits at baseband, it can be used for transmit or receive arrays. Programmable time delay only grants a second beam to control, but additional degrees of freedom for controlling the element phase response could be added.

While each approach has distinct disadvantages, a future multibeam paradigm could provide many full-power, electronically steerable, receive and transmit capable beams. Introducing additional degrees of freedom to the element phase response (building on the technique presented in this work) is a promising pathway to fully capable multibeam arrays.

\section{CONCLUSION}

This work describes the scalable router architecture and its implications. The scalable router is a decentralized relay 
array architecture, whose elements (branches) do not need shared timing or phase references to perform beamforming. TTD control within each branch is critical to ensure data coherence. Table II compares the presented branch circuit to other integrated TTD circuits (albeit intended for purposes other than a scalable router). From this branch circuit, a fully integrated four-branch radiative prototype was created and used to demonstrate data transmission with coherence correction, as well as control of two independent full power beams from the same array.

While the scalable router offers intriguing advantages, it bolsters rather than replaces existing systems. The scalable router naturally fits into emerging communication networks with smaller cells, higher frequencies, and line of sight issues and follows broader trends toward distributed systems. The scalable router's fully distributed and decentralized nature, adaptability, and capacity to operate as a single aperture spread across a variety of physically separated surfaces offer a valuable piece in the larger puzzle of future communication networks.

\section{ACKNOWLEDGMENT}

The authors would like to thank B. Abiri for his contribution to the integrated circuit digital interface and the members of the Caltech Holistic Integrated Circuits Lab for their help in determining a clear and complete approach for presenting this material.

\section{REFERENCES}

[1] M.-Y. Huang and H. Wang, "A mm-Wave wideband MIMO RX with instinctual array-based blocker/signal management for ultralow-latency communication," IEEE J. Solid-State Circuits, vol. 54, no. 12, pp. 3553-3564, Dec. 2019.

[2] S. Shahramian, M. J. Holyoak, A. Singh, and Y. Baeyens, "A fully integrated 384-element, 16-tile, $W$-band phased array with self-alignment and self-test," IEEE J. Solid-State Circuits, vol. 54, no. 9 , pp. 2419-2434, Sep. 2019.

[3] J. Pang et al., "A 28-GHz CMOS phased-array beamformer utilizing neutralized bi-directional technique supporting dual-polarized MIMO for 5G NR," IEEE J. Solid-State Circuits, vol. 55, no. 9, pp. 2371-2386, Sep. 2020

[4] A. Hajimiri, A. Komijani, A. Natarajan, R. Chunara, X. Guan, and H. Hashemi, "Phased array systems in silicon," IEEE Commun. Mag., vol. 42, no. 8, pp. 122-130, Aug. 2004.

[5] M. I. Skolnik, Radar Handbook, 2nd Ed. New York, NY, USA: McGraw-Hill, 1990.

[6] A. Sendonaris, E. Erkip, and B. Aazhang, "Increasing uplink capacity via user cooperation diversity," in Proc. IEEE Int. Symp. Inf. Theory, Aug. 1998, p. 156.

[7] A. Sendonaris, E. Erkip, and B. Aazhang, "User cooperation diversityPart I: System description," IEEE Trans. Commun., vol. 51, no. 11, pp. 1927-1938, Nov. 2003.

[8] A. Sendonaris, E. Erkip, and B. Aazhang, "User cooperation diversityPart II: Implementation aspects and performance analysis," IEEE Trans. Commun., vol. 51, no. 11, pp. 1939-1948, Nov. 2003.

[9] X. Tao, X. Xu, and Q. Cui, "An overview of cooperative communications," IEEE Commun. Mag., vol. 50, no. 6, pp. 65-71, Jun. 2012.

[10] J. N. Laneman, G. W. Wornell, and D. N. C. Tse, "An efficient protocol for realizing cooperative diversity in wireless networks," in Proc. IEEE Int. Symp. Inf. Theory, Jun. 2001, p. 294.

[11] A. Scaglione and Y.-W. Hong, "Opportunistic large arrays: Cooperative transmission in wireless multihop ad hoc networks to reach far distances," IEEE Trans. Signal Process., vol. 51, no. 8, pp. 2082-2092, Aug. 2003.
[12] V. Havary-Nassab, S. Shahbazpanahi, A. Grami, and Z.-Q. Luo, "Distributed beamforming for relay networks based on second-order statistics of the channel state information," IEEE Trans. Signal Process., vol. 56, no. 9, pp. 4306-4316, Sep. 2008.

[13] Y. Jing and H. Jafarkhani, "Network beamforming using relays with perfect channel information," IEEE Trans. Inf. Theory, vol. 55, no. 6, pp. 2499-2517, Jun. 2009.

[14] A. Dimas, D. S. Kalogerias, and A. P. Petropulu, "Cooperative beamforming with predictive relay selection for urban mmWave communications," IEEE Access, vol. 7, pp. 157057-157071, 2019.

[15] M.-K. Cho, I. Song, and J. D. Cressler, "A true time delay-based SiGe bi-directional T/R chipset for large-scale wideband timed array antennas," in Proc. IEEE Radio Freq. Integr. Circuits Symp. (RFIC), Jun. 2018, pp. 272-275.

[16] I. Mondal and N. Krishnapura, "A 2-GHz bandwidth, 0.25-1.7 ns truetime-delay element using a variable-order all-pass filter architecture in $0.13 \mu \mathrm{m} \mathrm{CMOS,"} \mathrm{IEEE} \mathrm{J.} \mathrm{Solid-State} \mathrm{Circuits,} \mathrm{vol.} \mathrm{52,} \mathrm{no.} \mathrm{8,}$ pp. 2180-2193, Aug. 2017.

[17] S. K. Garakoui, E. A. M. Klumperink, B. Nauta, and F. E. van Vliet, "Compact cascadable G M-C all-pass true time delay cell with reduced delay variation over frequency," IEEE J. Solid-State Circuits, vol. 50, no. 3, pp. 693-703, Mar. 2015.

[18] S. Jang, R. Lu, J. Jeong, and M. P. Flynn, "A 1-GHz 16-element four-beam true-time-delay digital beamformer," IEEE J. Solid-State Circuits, vol. 54, no. 5, pp. 1304-1314, May 2019.

[19] E. Ghaderi, A. Sivadhasan Ramani, A. A. Rahimi, D. Heo, S. Shekhar, and S. Gupta, "An integrated discrete-time delay-compensating technique for large-array beamformers," IEEE Trans. Circuits Syst. I, Reg. Papers, vol. 66, no. 9, pp. 3296-3306, Sep. 2019.

[20] W. Hong et al., "Multibeam antenna technologies for 5G wireless communications," IEEE Trans. Antennas Propag., vol. 65, no. 12, pp. 6231-6249, Dec. 2017.

[21] T.-S. Chu and H. Hashemi, "True-time-delay-based multi-beam arrays," IEEE Trans. Microw. Theory Techn., vol. 61, no. 8, pp. 3072-3082, Aug. 2013.

[22] T. Nishio, H.-P. Tsai, Y. Wang, and T. Itoh, "A high-speed adaptive antenna array with simultaneous multibeam-forming capability," IEEE Trans. Microw. Theory Techn., vol. 51, no. 12, pp. 2483-2494, Dec. 2003.

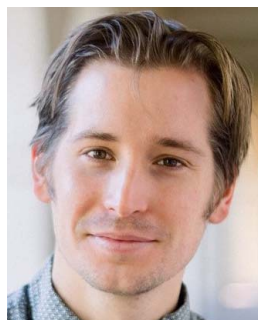

Austin Fikes (Graduate Student Member, IEEE) received the B.S. degree in engineering from the Harvey Mudd College, Claremont, CA, USA, in 2016, and the M.S. degree in electrical engineering from the California Institute of Technology (Caltech), Pasadena, CA, USA, in 2018, where he is currently pursuing the Ph.D. degree at the Caltech Holistic Integrated Circuit Lab.

His research focuses on decentralized, large-scale, microwave arrays with novel form factors.

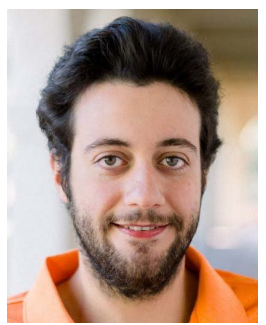

Parham P. Khial (Graduate Student Member, IEEE) was born in Tehran, Iran, in 1993. He received the B.S. degree in electrical engineering from the Sharif University of Technology, Tehran, in 2015, and the M.S. degree in electrical engineering from the California Institute of Technology (Caltech), Pasadena, CA, USA, in 2016, where he is currently pursuing the Ph.D. degree in electrical engineering under the supervision of Prof. Hajimiri.

His current research interests include millimeter-wave and radio frequency integrated circuits for biomedical and communications applications, and silicon photonics platforms for the next generation of optical sensors.

Mr. Khial was a recipient of the Gold Medal winner of the National Astronomy Olympiad in Iran, in 2010, the Bronze and Silver Medal Winner of the 5th International Olympiad on Astronomy and Astrophysics (5th IOAA) in Poland, in 2011, and the Analog Devices Outstanding Student Designer Award in 2016. 


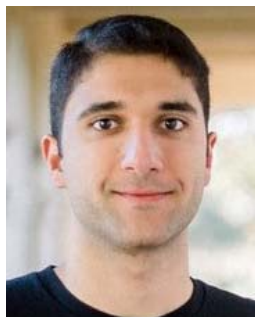

Samir Nooshabadi (Graduate Student Member, IEEE) received the B.S. degree in electrical engineering from the University of Michigan, Ann Arbor, MI, USA, in 2019. He is currently pursuing the Ph.D. degree at the Caltech Holistic Integrated Circuit Lab, California Institute of Technology, Pasadena, CA, USA.

His research interests are in high-frequency integrated circuits and systems.

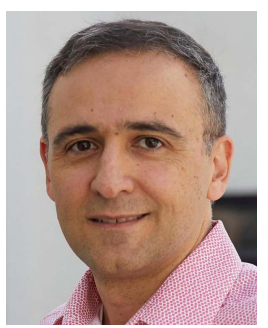

Ali Hajimiri (Fellow, IEEE) received the B.S. degree in electronics engineering from the Sharif University of Technology, Tehran, Iran, in 1994 and the M.S. and Ph.D. degrees in electrical engineering from Stanford University, Stanford, CA, USA, in 1996 and 1998, respectively.

He was with Philips Semiconductors, Sunnyvale, CA, where he worked on a BiCMOS chipset for GSM and cellular units from 1993 to 1994. In 1995, he was with Sun Microsystems, Sunnyvale, working on the UltraSPARC microprocessors cache RAM design methodology. In summer 1997, he was with Lucent Technologies (Bell Labs), Murray Hill, NJ, USA, where he investigated low-phase-noise integrated oscillators. In 1998, he joined the Faculty of the California Institute of Technology, Pasadena, CA, USA, where he is currently the Bren Professor of Electrical Engineering and Medical Engineering, the Director of the Microelectronics Laboratory, and the Co-Director of the Space Solar Power Project. In 2002, he cofounded Axiom Microdevices Inc., Irvine, CA, whose fully integrated CMOS PA has been shipped around 400000000 units, and was acquired by Skyworks Inc., Irvine, in 2009. He is also a cofounder of
GuRu Wireless Inc., Pasadena, CA, He is the author of Analog: Inexact Science, Vibrant Art (Early Draft, 2021), a book on fundamental principles of analog circuit design, and "The Design of Low Noise Oscillators" (Boston, MA: Springer). He has authored and coauthored more than 250 refereed journal and conference technical articles. He holds more than 120 U.S. patents with many more pending applications. His research interests are high-speed and high-frequency integrated circuits for applications in sensors, photonics, biomedical devices, and communication systems.

Prof. Hajimiri is also a Fellow of the National Academy of Inventors (NAI) He was selected to the TR35 Top Innovator's List. He was a recipient of the Microwave Prize. He won the Feynman Prize for Excellence in Teaching, Caltech's most prestigious teaching honor, Caltech's Graduate Students Council Teaching and Mentoring Award, and the Associated Students of Caltech Undergraduate Excellence in Teaching Award. He was the Gold Medal Winner of the National Physics Competition and the Bronze Medal Winner of the 21st International Physics Olympiad, Groningen, The Netherlands. He was recognized as one of the top-ten contributors to the International Solid-State Circuits Conference (ISSCC). He was a co-recipient of the IEEE Journal of Solid-State Circuits Best Paper Award, the ISSCC Jack Kilby Outstanding Paper Award, the RFIC Best Paper Award, the IMS Best Advance Practices Awards, a two-time co-recipient of the CICC Best Paper Award, and a three-time winner of the IBM Faculty Partnership Award, the National Science Foundation CAREER Award, and the Okawa Foundation Award. $\mathrm{He}$ has served as a Distinguished Lecturer of the IEEE Solid-State and Microwave Societies. He has served on the Technical Program Committee of the International Solid-State Circuits Conference (ISSCC), as an Associate Editor of the IEEE JOURNAL OF SOLID-STATE CIRCUITS (JSSC) and IEEE TRANSACTIONS ON CIRCUITS AND SYSTEMS (TCAS) II, a member of the Technical Program Committees of the International Conference on Computer Aided Design (ICCAD), a Guest Editor of the IEEE TRANSACTIONS ON Microwave Theory And Techniques, and a Guest Editorial Board of the Transactions of Institute of Electronics, Information and Communication Engineers of Japan (IEICE). 